\title{
Direct observation of liquid crystals using cryo-TEM: specimen preparation and low-dose imaging
}

\begin{tabular}{|c|c|}
\hline Journal: & Microscopy Research and Technique \\
\hline Manuscript ID: & MRT-14-042.R1 \\
\hline Wiley - Manuscript type: & Research Article \\
\hline Date Submitted by the Author: & $\mathrm{n} / \mathrm{a}$ \\
\hline Complete List of Authors: & $\begin{array}{l}\text { Gao, Min; Kent State University, Liquid Crystal Institute } \\
\text { Kim, Young Ki; Kent State university, Liquid crystal institute } \\
\text { Zhang, Cuiyu; Kent State University, Liquid Crystal Institute } \\
\text { Borshch, Volodymyr; Kent State University, Liquid Crystal Institute } \\
\text { Zhou, Shuang; Kent State University, Liquid Crystal Institute } \\
\text { Park, Heung-Shik; Kent State University, Liquid Crystal Institute } \\
\text { Jákli, Antal; Kent State University, Liquid Crystal Institute } \\
\text { Lavrentovich, Oleg; Kent State University, Liquid Crystal Institute } \\
\text { Tamba, Maria-Gabriela; University of Hull, Department of Chemistry } \\
\text { Kohlmeier, Alexandra; University of Hull, Department of Chemistry } \\
\text { Mehl, Georg; University of Hull, Department of Chemistry } \\
\text { Weissflog, Wolfgang; Martin Luther University Halle-Wittenberg, } \\
\text { Department of Chemistry and Physical Chemistry } \\
\text { Studer, Daniel; University of Bern, Institute of Anatomy } \\
\text { Zuber, Benoit; University of Bern, Institute of Anatomy } \\
\text { Gnägi, Helmut; Diatome Ltd Switzerland, } \\
\text { Lin, Fang; South China Agricultural University, College of Science }\end{array}$ \\
\hline Keywords: & freeze fracture, high pressure freezing, CEMOVIS, thermotropic, lyotropic \\
\hline
\end{tabular}


Direct observation of liquid crystals using cryo-TEM: specimen preparation and low-dose imaging

Min Gao, ${ }^{1}$ * Young-Ki Kim, ${ }^{1}$ Cuiyu Zhang, ${ }^{1}$ Volodymyr Borshch, ${ }^{1}$ Shuang Zhou, ${ }^{1}$ Heung-Shik Park, ${ }^{1}$ Antal Jákli, ${ }^{1}$ Oleg D. Lavrentovich, ${ }^{1}$ Maria-Gabriela Tamba, ${ }^{2}$ Alexandra Kohlmeier, ${ }^{2}$ Georg H. Mehl, ${ }^{2}$ Wolfgang Weissflog, ${ }^{3}$ Daniel Studer, ${ }^{4}$ Benoît Zuber, ${ }^{4}$ Helmut Gnägi, ${ }^{5}$ and Fang $\operatorname{Lin}^{6}$

${ }^{1}$ Liquid Crystal Institute and Chemical Physics Interdisciplinary Program, Kent State University, Kent, $\mathrm{OH} 44242$, USA

${ }^{2}$ Department of Chemistry, University of Hull, Hull, HU6 7RX, UK

${ }^{3}$ Department of Chemistry and Physical Chemistry, Martin Luther University Halle-Wittenberg, von-Danckelmann-Platz 4, 06120 Halle, Germany

${ }^{4}$ Institute of Anatomy, University of Bern, Baltzerstrasse 2, 3000 Bern 9, Switzerland

${ }^{5}$ Diatome Ltd Switzerland, P.O. Box 557, CH-2501 Biel, Switzerland

${ }^{6}$ College of Science, South China Agricultural University, Guangzhou, Guangdong 510642, China

Key words: freeze fracture, high pressure freezing, CEMOVIS, thermotropic, lyotropic

Running title: TEM of liquid crystals: specimen preparation and low-dose imaging

*Correspondence to: Min Gao, Liquid Crystal Institute, Kent State University, 1425 University Esplanade, Kent, OH 44242. Email: mgao@kent.edu, office phone: 330-672-7999 


\begin{abstract}
Liquid crystals (LCs) represent a challenging group of materials for direct transmission electron microscopy (TEM) studies due to the complications in specimen preparation and the severe radiation damage. In this paper, we summarize a series of specimen preparation methods, including thin film and cryo-sectioning approaches, as a comprehensive toolset enabling highresolution direct cryo-TEM observation of a broad range of LCs. We also present comparative analysis using cryo-TEM and replica freeze-fracture TEM on both thermotropic and lyotropic LCs. In addition to the revisits of previous practices, some new concepts are introduced, e.g., suspended thermotropic LC thin films, combined high pressure freezing and cryo-sectioning of lyotropic LCs, and the complementary applications of direct TEM and indirect replica TEM techniques. The significance of subnanometer resolution cryo-TEM observation is demonstrated in a few important issues in LC studies, including providing direct evidences for the existence of nanoscale smectic domains in nematic bent-core thermotropic LCs, comprehensive understanding of the twist-bend nematic phase, and probing the packing of columnar aggregates in lyotropic chromonic LCs. Direct TEM observation opens ways to a variety of TEM techniques, suggesting that TEM (replica, cryo, and in situ techniques), in general, may be a promising part of the solution to the lack of effective structural probe at the molecular scale in LC studies.
\end{abstract}




\section{Introduction}

Transmission electron microscopy (TEM) techniques are under fast development in terms of, e.g., resolving power (Meyer et al., 2008), temporal resolution (Kim et al., 2008), and in situ environmental capability (Gai and Boyes, 2009). However, for many real-world problems, the outputs (for example, imaging resolution) are often limited by the material properties rather than by the microscope performance. On the other hand, the technical developments in microscopyrelated fields enable us to revisit some of the earlier challenging problems and get much improved results. In this paper, we report some of the initial results of our on-going efforts to repursue high resolution direct TEM imaging of liquid crystals (LCs), a group of materials having tremendous impact (e.g., liquid crystal display, the so-called LCD) but imposing great challenges for direct TEM observation.

LCs uniquely combine order and mobility, and are generally described as intermediate states (mesophases) of matter between crystalline solid (possessing both orientational and 3D positional orders) and liquid (with neither orientational nor positional orders) (de Gennes and Prost, 1995; Collings, 2002). Organic LCs can be divided into thermotropic and lyotropic LCs, with the phase transition (change in the amount of order) mainly driven by temperature in the former, and by concentration or percentage of the added solvent (most often, water) in the latter. A typical single component thermotropic LC consists of molecules (mesogens) with, for example, rigid rod-like or sometimes disk-like middle part and attached flexible ends. Thermotropic LC materials may exhibit one or more mesophases (most commonly nematics and smectics) between the high temperature isotropic liquid and the low temperature crystalline phases. Simply speaking, a nematic phase has only orientational order but no long-range positional order. In the best known uniaxial nematics, the randomly positioned molecules align favorably along a single axis, the so-called director $\hat{n}$. In addition to the orientational order, a smectic phase also has a degree of positional order, as the molecules tend to form layers stacked on top of each other. There is no further positional order inside each layer for the two common smectic phases: smectic A (SmA), in which the rod-like molecules are parallel to the layer normal, and smectic C $(\mathrm{SmC})$, in which the molecules are tilted with respect to the layer normal. While for a smectic B $(\mathrm{SmB})$ phase, the molecules, orienting on average along the layer normal, are positioned in a hexagonal lattice within the layers. Details on the structure of other smectic and crystalline-like mesophases can be found elsewhere (de Gennes and Prost, 1995).

Lyotropic LCs are composed of mixed LC molecules and solvent. The most well-known lyotropic LCs are formed by amphiphiles, i.e., molecules with one hydrophilic "head" and one or two hydrophobic ends. Representative examples are soaps, phospholipids, and surfactants. 
Driven by hydrophobic/hydrophilic interactions, the amphiphilic molecules in solvent (usually water) aggregate into vesicles and micelles. At sufficient concentrations, the aggregates of amphiphilic molecules of different shapes pack into mesophases such as lamellar, hexagonal columnar and cubic phases depending mainly on the concentration and properties of the hydrophobic and hydrophilic molecular parts. A distinct but broad family of lyotropic LCs is formed by chromonic LCs, consisting of mesogens with disk-like aromatic central core and ionic outer groups (Park and Lavrentovich, 2012). While in water, the molecules self-assemble into columnar aggregates by face-to-face stacking that minimizes the contact area with water. Mainly two LC phases can be formed by these self-assembled columnar aggregates: a low concentration nematic phase and a high concentration hexagonal columnar phase, often called "M phase" (Lydon, 2010). Since the molecules in aggregates are bound together by weak non-covalent interactions, the length of the aggregates depends strongly on temperature. Thus the phase diagrams of chromonic LCs, unlike their amphiphilic lyotropic counterparts, are very sensitive to the temperature (Park and Lavrentovich, 2012).

The understanding of detailed LC behaviors at the molecular level is surprisingly limited, which can be partly attributed to their uniquely complicated structure and the lack of effective structural probe at nanometer and subnanometer scale. Beyond the classic polarized light microscopy (PLM) characterizing structures at a scale of micrometers, x-ray diffraction, including synchrotron small-angle $x$-ray scattering (SAXS), has been the most widely used nanoscale structural characterization tool in LCs (Hong et al., 2010), targeting mainly ordered structures with periods less than tens of nanometers. Nanoscale imaging techniques, e.g., TEM, scanning electron microscopy (SEM) (Rizwan et al., 2007), scanning tunneling microscopy (Frommer, 1992), and atomic force microscopy (Yashima, 2010), have also been used, though their applications so far have been relatively limited. Among the imaging techniques, a replica TEM technique, namely freeze fracture TEM (FFTEM), applied to LCs since 1970's (Lydon and Robinson, 1972; Kléman et al., 1977; Costello et al., 1984), has attracted continuous interests by revealing the internal structures of a variety of LC materials (Hough et al., 2009; Chen et al., 2013; Borshch et al., 2013).

Two major challenges exist for the successful application of direct TEM imaging in LC studies: preparing TEM specimens with preserved native structure and minimizing radiation damage during TEM observation. The LC materials are similar to organic liquids, in which the small molecules (typically a few nanometers long) are moving rather freely and spend only a limited time at the preferred orientation. The forces keeping the orientational order are very weak, which makes it easy to use electrical or magnetic field to manipulate the overall director for practical applications, but also leads to a relatively high sensitivity to electron beam as compared even to typical biomaterials. For TEM specimen preparation, LCs also pose some unique challenges in 
addition to the problems shared by many non-solid materials. At the current stage, it is common to use cryo-TEM to observe plunge-frozen thin films of fluid-like materials that are electron transparent. However, the molecular alignment in LCs is highly sensitive to the presence of surfaces and interfaces. The reason is that the surfaces and interfaces introduce aligning forces acting on the LC bulk, thanks to the anisotropy of surface interaction (the so-called surface anchoring phenomenon) (Jérôme, 1991). As a result, thin specimens may show structures strongly influenced by the surface forces. Some surface-induced structures can be very different from the bulk LC structures, as shown in the examples of the so-called helical nanofilament phase in contact with glass substrate (Chen et al., 2012) and the nematic LC that develops smectic layering near the surface (Ruths et al., 1996). In addition, the surface anchoring may set the molecular orientation in a direction (for example, parallel to the electron beam) that is difficult for TEM observation. On the other hand, to freeze a thick sample with minimized influence of the surface forces is often not practical, because of the insufficient cooling rate. The frozen thick samples may fail to preserve the native structure, which is especially true for lyotropic LCs with high water content and some high temperature thermotropic phases.

Due to the above challenges, despite the promising early direct TEM imaging results (VoigtMartin and Durst, 1987; Hara et al., 1988; Gilli et al., 1991; Voigt-Martin et al., 1992; Bunning et al., 1994), it is the indirect technique of FFTEM that remains to be a dominant high resolution imaging tool for LC bulk structures nowadays. In FFTEM, a frozen LC sample is first fractured at low temperature in vacuum, and then the fractured surface is replicated by a thin deposition of heavy metal at an angle to create shadows of the surface topography, followed by a continuous carbon supporting layer deposited from the top. The resolution of the FFTEM is largely determined by the particle size of the deposited heavy metal, which is normally a few nanometers. Although FFTEM effectively avoids radiation damage by using replica specimens, it disables the use of many TEM techniques (e.g., diffraction and spectroscopy), and has a very limited potential towards sub-nanometer imaging which is essential to understand LC behaviors (phase transition, defect, interfaces, etc.) at the molecular scale.

In this paper, we demonstrate a series of specimen preparation routines for LCs, aiming to set up an easily accessible toolbox for TEM imaging especially direct cryo-TEM examination of the majority of thermotropic and lyotropic LC phases. In addition to adopting and modifying a few of the previous practices (Pierron et al., 1995; Kostko et al., 2005), some new concepts are introduced, e.g., suspended thermotropic LC thin films, cryo-sectioning of high pressure frozen lyotropic LCs, and complementary combination of direct cryo-TEM and replica FFTEM. For sub-nanometer resolution imaging, we demonstrate that a widely available cryo-TEM, a high sensitivity CCD camera and a modified low-dose imaging procedure have made a ready combination for many challenging LC materials. We present examples of applying the above 
techniques to reveal the presence of nanoscale smectic clusters in bent-core nematic LCs, the existence of twist-bend nematic mesophase with a complex director structure that follows the geometry of an oblique helicoid with a nanoscale pitch, and nematic arrangement of the elongated aggregates in the lyotropic chromonic LCs. Direct TEM imaging opens the door for applying a variety of TEM techniques beyond low-dose imaging, which makes TEM a promising molecular scale structural probe for LC and other soft-matter materials.

\section{Materials and Methods}

\section{LC materials}

Figure 1 shows the chemical structure of the LC molecules discussed in this paper. The formal names and their short forms are both given. We report on the microstructure of three thermotropic (namely, 3RBC-S, 3RBC-N, and CB7CB) and two lyotropic chromonic (DSCG and SSY) LCs. The two three-ring bent-core (3RBC) LCs, are formed by banana-like molecules, in which a bent rigid core has two aliphatic flexible chains attached to its ends (Weissflog et al., 2012). The $3 \mathrm{RBC}-\mathrm{S}$ forms a SmA phase, while the $3 \mathrm{RBC}-\mathrm{N}$ forms a nematic phase. In the case of dimeric thermotropic mesogen $\mathrm{CB} 7 \mathrm{CB}$, the molecule represents two rigid rod-like segments connected by a flexible central aliphatic bridge (Panov et al., 2010). As representatives of chromonic LCs, we used the two most widely studied mesogens (Park and Lavrentovich, 2012): disodium cromoglycate (DSCG), known also as an anti-asthmatic drug, and Sunset Yellow FCF (SSY), a food dye. The DSCG samples used here have concentrations of $6.2 \mathrm{wt} \%(0.129 \mathrm{~mol} / \mathrm{kg})$ and $15 \mathrm{wt} \%(0.344 \mathrm{~mol} / \mathrm{kg})$ in water, determined by PLM to be in isotropic and nematic phases at room temperature, respectively (Kim et al., 2013). The nematic SSY sample is $30.7 \mathrm{wt} \%$ $\left(0.98 \mathrm{~mol} / \mathrm{kg}\right.$ ) with $64.8 \%$ water and $4.5 \% \mathrm{MgSO}_{4}$ (Zhou et al., 2012). In addition, we show results on Au nanoparticle doped 5CB.

\section{TEM specimen preparation procedures}

Figure 2 is a schematic overview showing the basic routines used in this study to prepare TEM specimens of thermotropic (Fig. 2a-2c) and lyotropic (Figs. 2d-2f) LCs for cryo-TEM and FFTEM. Rapid freezing is used in all the routines to quench the flowing LC materials, making the static structure inside a TEM specimen basically a snapshot of the LC material with both orientational and positional fluctuations. Here we categorize the techniques into "thin film" approach and "bulk" approach based on the form of the pre-freezing sample. In the former, the LC material or at least part of it is already electron transparent and can be transferred directly into the TEM after the freezing process. While for the "bulk" approach, much thicker (a few microns to hundreds of microns) material is first frozen and then goes through further processing 
to obtain electron transparent TEM specimen. The detailed procedures of the methods used in this study are described as follows.

1) Thin film approach: plunge freezing of thermotropic and lyotropic LCs

For thermotropic LCs, we employed a simple but effective procedure to obtain LC thin films either suspended or supported by thin carbon film. As shown in Fig. 2a, the LC materials were first dissolved in an evaporable solvent (most often chloroform in this study) to make a $\sim 0.1$ $0.3 \%$ solution. A few microliters of the solution were put on carbon (continuous or holy) coated TEM grids. The grids are normally treated with oxygen plasma in a Fischione 1020 plasma cleaner using 25\% oxygen and 75\% Ar mixture gas. LC thin films were deposited on the carbon films after the solvent evaporates, while suspended films were obtained in the holes of holey carbon films. The thin films (together with the TEM grids) then underwent thermal treatment to achieve the desired phase, followed by a fast plunge freezing into liquid ethane or liquid nitrogen to preserve the structure of the desired mesophase. The thermal treatment normally includes heating to the isotropic phase, and cooling down to the target temperature. To achieve repeatable results, enough time should be allowed for the specimen to stabilize at each temperature and during the heating/cooling process. The thickness of the films can be controlled by the total volume of the LC solution put on the grids and the concentration of the solution, but normally a large variation in film thickness can be seen within the same TEM specimen. It is also recommended to check the LC thin films using PLM with a heating stage to make sure that the thin films still exhibits the basic characteristics of the LC materials, especially for multicomponent materials.

For lyotropic LCs with a high percentage of water $(>80 \%)$, it is a straightforward process to prepare thin film cryo-TEM specimens using plunge freezing due to their relatively low viscosity (Fig. 2d). In this study, we used a FEI Mark-IV Vitrobot to plunge-freeze the lyotropic samples at room temperature and $\sim 100 \%$ humidity. Less than $3 \mu \mathrm{l}$ of the lyotropic LC solution was put on a lacey carbon coated TEM grid and blotted by two filter papers, followed by plunge vitrification in liquid ethane.

\section{2) Bulk approach: cryo-sectioning of thermotropic and lyotropic LCs}

In this study, cryo-sectioning was utilized to prepare TEM specimens from frozen LC materials (CB7CB, DSCG, and SSY). As shown in Figs. 2b and 2e, the thermotropic and lyotropic LCs were first quenched using plunge freezing (in liquid nitrogen or ethane) and high-pressure freezing (Leica EM PACT2), respectively. The following cryo-sectioning was implemented at $150^{\circ} \mathrm{C}$ or $-160^{\circ} \mathrm{C}$ using a Diatome cryo $25^{\circ}$ diamond knife mounted on Leica UC7/FC7 cryoultramicrotome. The nominal slice thickness was set to $50-80 \mathrm{~nm}$. The sections were transferred 
onto holey lacey carbon coated copper grids. In the high pressure freezing of lyotropic LCs, the LC solution was filled into copper capillary tubes of $\sim 300 \mu \mathrm{m}$ in inner-diameter. A high pressure ( $\sim 2000$ bar) was applied at the two ends of the tube to dramatically slow down the crystallization of water. Immediately after the pressure application, liquid nitrogen was injected onto the tube to freeze the sample (Studer et al., 2001). For the SSY sample containing 64.8\% water, no cryoprotectant was used in the vitrification. While for the DSCG samples with $85 \%$ and $93.8 \%$ water, $10-20 \%$ of dextran $\left(\mathrm{M}_{\mathrm{r}} \sim 70 \mathrm{k}\right)$ was added to vitrify the solutions successfully.

\section{3) Bulk approach: replica FFTEM}

In this study, we also employ FFTEM to study thermotropic CB7CB and lyotropic DSCG. For CB7CB, the LC material was put between two copper planchettes (Fig. 2c). The sandwich structure was then stabilized at $125^{\circ} \mathrm{C}$ (isotropic phase), and cooled down to $95^{\circ} \mathrm{C}$ to obtain the low temperature twist-bend nematic phase (Borshch et al., 2013). After quenching in liquid nitrogen, the assembly was quickly transferred into the vacuum chamber of a freeze-fracture apparatus (BalTec BAF060) with the sample stage temperature set at $-140^{\circ} \mathrm{C}$. Inside the chamber, a built-in microtome was used to break the assembly and expose the fracture surface. $\sim 4 \mathrm{~nm}$ thick $\mathrm{Pt} / \mathrm{C}$ was then deposited onto the fractured surface at a $45^{\circ}$ angle to create shadowing of the surface morphology, followed by a $\sim 20 \mathrm{~nm}$ thick $\mathrm{C}$ deposition from the top to form a continuous supporting film. The samples were then warmed up and removed from the freeze fracture chamber. The LC material was dissolved in chloroform, while the replica film was collected and placed onto carbon coated TEM grid. For DSCG, the LC solution was vitrified using high pressure freezing (Fig. 2f), and fractured at $-160^{\circ} \mathrm{C}$. In Fig. $2 \mathrm{f}$, we also show schematically the widely used plunge freezing routine to prepare FFTEM specimen from lyotropic LCs, in which a thin layer of the LC solution (a few microns thick) is placed between two copper sheets.

In our practice, complementary use of the above routines has been applied to both thermotropic and lyotropic LCs, which helps towards comprehensive understanding of the complicated LC behaviors. In this paper, we report results of combined thin film plunge freezing, bulk cryosectioning, and bulk FFTEM, on two of the samples (CB7CB and DSCG).

\section{TEM observation and a modified Cryo-TEM low-dose procedure}

TEM results shown in this paper were obtained using a versatile FEI Tecnai F20 microscope (200 kV) equipped with scanning TEM unit, energy-dispersive x-ray spectrometer (EDS), Gatan imaging filter, anti-contaminator, and low-dose operation mode. A Gatan 626.DH cryo-holder was used to keep the specimen temperature $<-170^{\circ} \mathrm{C}$ for cryo-TEM, while the replica specimens of FFTEM were examined at room temperature. The TEM images were recorded using a Gatan UltraScan 4K CCD camera. 
A modified cryo-TEM low dose procedure was employed in this study especially for thermotropic LCs in which the microstructure often cannot be visualized with large underfocus at low magnification. In the modified procedure, the electron beam was carefully aligned to maintain the same position and size for different "spot sizes" (corresponding to the current of the $1^{\text {st }}$ condenser lens) even for high magnification ( $70 \mathrm{~K}-90 \mathrm{~K}$ in our study). The searching and focusing of the area of interest (AOI) were carried out quickly at the same magnification as the final exposure, but at a much lower beam intensity/area controlled directly by "spot size". The low-magnification searching was only used to locate regions in the LC films with suitable thickness. To minimize radiation damage, the beam was always blanked when the specimen was not imaged. Compared to the dose level used for normal biomaterials $\left(10-25 \mathrm{e}^{-1} / \AA^{2}\right)$, a much smaller dose level was normally required for thermotropic LCs. For some extremely sensitive materials, a dose of $0.2 \mathrm{e}^{-/ \AA^{2}}$ was often used for the rapid searching, and $2 \mathrm{e}^{-} / \AA^{2}$ for the image acquisition. The movement of specimen and underfocus can help the searching of AOIs at low dose. It should be also pointed out that some LC materials are relatively more robust under electron beam than others, and a higher dose can be applied to improve the signal/noise ratio. On the other hand, the standard low-dose procedure works well for lyotropic LCs which normally have uniform structure and have comparable beam sensitivity to aqueous biological samples.

To study the influence of surface effect and film thickness, low-loss electron energy-loss spectroscopy (EELS) was used to determine the inelastic mean-free-path (MFP) and the thickness of the LC thin films (Egerton, 2011). The MFPs measured from several thermotropic LCs materials range between $130 \mathrm{~nm}$ and $150 \mathrm{~nm}$.

\section{Results and Discussion}

\section{Subnanometer resolution imaging of thermotropic and lyotropic LCs using thin film approach}

For a beam sensitive material, the achievable resolution in a given high-performance TEM is mainly limited by statistical noise (depending on the electron dose level) and the property of the image recording device. Our measurements (not shown) on the Gatan Ultrascan 4000 CCD camera (Zhang et al., 2003) used in this study indicate that a dose rate of $\sim 10 \mathrm{e}^{-} /$pixel or even less can allow an imaging resolution of 2-3 pixels (considering the Nyquist theorem for 2dimensional imaging). For example, at $\sim 10 \mathrm{e}^{-} /$pixel and a magnification of $285 \mathrm{~K}$ (binning $2,0.78$ $\AA /$ pixel), the $2.04 \AA \mathrm{Au}$ (200) lattice can be resolved, which is 1.3 times the Nyquist frequency spacing (1.56 $\AA$ ). Using this as a rough guidance, we can estimate that for our routinely used imaging condition (a magnification of $71 \mathrm{~K}$ at a binning of 2 for the $\mathrm{CCD}$, leading to a pixel size of $3.3 \AA /$ pixel) and the minimum dose of $2 \mathrm{e}^{-/} \AA^{2}$ ( $22 \mathrm{e}^{-/ p i x e l ~ w i t h o u t ~ t h e ~ s p e c i m e n), ~ a ~}$ subnanometer resolution can be achieved for thin specimens. A higher resolution and "nice" 
images to bare eyes (with better signal/noise ratio) can be obtained for those more robust samples to which higher dose can be applied. It should be pointed out that the results demonstrated in this paper were taken mostly from materials that can stand a higher dose.

We have employed such "high resolution" direct imaging to study the nanoscale structure of the two 3RBC thermotropic LCs (Fig. 1), revealing the first direct evidence of smectic nanoclusters in the nematic phase of 3RBC-N (Zhang et al., 2012). The two molecules, 3RBC-S and 3RBC-N, have similar structure and length with rigid aromatic cores and flexible hydrocarbon tails. 3RBC$\mathrm{S}$ has a macroscopic SmA mesophase in the temperature range between $66.5^{\circ} \mathrm{C}$ and $118.6^{\circ} \mathrm{C}$. Fig. $3 \mathrm{a}$ is a cryo-TEM image from a 3RBC-S LC thin film quenched at SmA temperature range $\left(87^{\circ} \mathrm{C}\right.$ in this study), showing domains (numbered in Fig. 3a for convenience) separated by abrupt boundaries. The domains, ranging from a few hundreds of nanometers to microns in size, are believed to consist of smectic layers in different orientations, while the domain boundaries are corresponding to the discontinuity of the layers. The contrast of the domains may come from the differences in layer orientation and thickness. The domains (I, III, and V in Fig. 3a) with visible layered structure are those at edge-on orientation. The layers inside each domain are basically continuous, but often bent (Fig. 3a). Though the curvature of the layers is gradual, the orientation change can amount to a large degree with distance. Despite of the curvature, the layer spacing is very well defined, ranging between $3.75 \mathrm{~nm}$ and $3.85 \mathrm{~nm}$, as measured from the images and the fast Fourier transform (FFT) patterns (the inset of Fig. 3a). The result matches the $\sim 3.7 \mathrm{~nm}$ spacing determined by synchrotron SAXS (Zhang et al., 2012). Fig. 3b is a magnified image of the marked region in Fig. 3a, showing the smectic layers and the slight bend. A molecular model of the layered structure is given in the inset of Fig. 3b. The shape of the domains can be irregular. For example, judged by the layer continuity and orientation, domain I extends to the lower right region in Fig. 3a and is overlapped with domain III in the thickness direction. Fig. 3c shows a magnified image of the overlapping area (I+III), and the corresponding FFT pattern (the inset).

In contrast to $3 \mathrm{RBC}-\mathrm{S}, 3 \mathrm{RBC}-\mathrm{N}$ does not have a macroscopic smectic mesophase, but a nematic phase between $62.5^{\circ} \mathrm{C}$ and $100.1^{\circ} \mathrm{C}$. However, recently there has been speculation of the possible existence of short-range smectic-C clusters which may likely be the cause of some unusual macroscopic behavior in bent-core nematics (Hong et al., 2010). By quenching nematic 3RBC-N thin films at different temperatures during the cooling process from isotropic phase, we reported the observation of nanometer-sized smectic domains, which was enhanced at lower temperature (Zhang et al., 2012). Fig. 4a shows a typical cryo-TEM image and the corresponding FFT pattern from the $3 R B C-N$ thin films, indicating no long range $1 \mathrm{D}$ order but layered smectic nanoclusters in the nematic substrate. The smectic clusters are normally a few tens of nanometer in length and consist of 1 to 10 layers. The layer spacing of the clusters is not rigid and mostly varies between 
$2.8 \mathrm{~nm}$ and $3.1 \mathrm{~nm}$, which is independent of the quenching temperature. The considerably smaller spacing when compared to $3 \mathrm{RBC}-\mathrm{S}$ can be considered as an evidence for a tilted director, i.e., the formation of a SmC structure, as proposed by Hong et al. (2010). Similar to the abovementioned result for 3RBC-S, the layers can also bend, especially in larger clusters, and overlapping of clusters in the thickness direction can be observed (as indicated by solid arrows in Fig. 4a). The existence of smectic nanoclusters in the nematic phase, including well-defined single layers, indicates clearly the strong tendency of bent-core LC molecules to close pack into layers. Though very different orientations can be found in neighboring clusters due to the local director fluctuation (Zhang et al., 2012), notable orientation preference can be identified frequently in local areas, probably owing to the consistent director alignment as the smectic clusters evolve from the nematic substrate. Fig. $4 \mathrm{~b}$ shows a local region with the majority of the clusters having similar orientation. Figure $4 \mathrm{c}$ shows that some clusters with almost the same orientation are assembled into a large cluster with more than 40 layers. Such large assemblies can only be found rarely, but small scale side-by-side assemblies often with very similar orientations seem to be a common characteristics of the smectic nanoclusters. In Fig. 4a, we use hollow arrows to point out a few of them. The lower insets of Fig. 4a are magnified images of two assemblies enclosed by dashed squares. An edge dislocation-like extra half layer can be identified in the left inset. While in the right inset, a clear gap of a few nanometers in width can be seen in the lower part of the assembly where the layers of the two sides are not aligned along the layer normal, i.e., out of phase.

Besides the simple cases shown above, complicated situations also present frequently, leading to more complex assembly structures. For example, some neighboring clusters have different layer numbers or different orientations, and some assemblies involve more than two clusters. Figures $4 \mathrm{~d}-4 \mathrm{f}$ show a few representative complex assemblies. By using the snapshot specimens, it is difficult to determine the lifetime of the smectic clusters or the sequence and dynamics of the processes. However, the detailed structure revealed by direct TEM imaging, e.g., partially connected assemblies and adjustments of the layers near the cluster joints, seems to suggest that the molecules between the side-by-side clusters may align themselves in layers and form junctions to connect the neighboring clusters. Such later-stage "bridging" process may account for a major part of the complex assembly structures in addition to possible growth defects in individual clusters. Figure $4 \mathrm{~d}$ shows probably a fully connected assembly consisting of at least three small clusters which are roughly "in phase". The upper junction (marked by a hollow arrow) is smooth, while the lower one bends sharply because the connections of layers shift by one layer along the layer normal, i.e., the smectic layers in the junction have a different orientation from that of the clusters. The upper junction marked in Fig. 4e is between two parallel clusters shifted by half of the layer spacing, while the lower junction involves three clusters. Note that the two junctions are only 1-3 nanometers in width, even narrower than the layer spacing, and the left 
side of the lower junction seems to be incomplete, i.e., the alignment of the molecules in the gap may be still in progress before the freezing process. In addition, a short extra layer (pointed out by a solid arrow) is formed between the two upper clusters that tilt from each other by about $18^{\circ}$. Edge dislocation-like additional layers can be found when misalignments occur between the layers in different clusters, which is especially common when the two clusters have different layer numbers or orientations. Figure $4 \mathrm{f}$ shows another junction of three clusters. An extra half layer is indicated in cluster I as it forms a sharp bend with cluster III that is tilted by $\sim 40^{\circ}$ from cluster I. Though there is only $\sim 15^{\circ}$ tilt between clusters II and III, two extra half layers can still be identified (Fig. 4f). Figure $4 \mathrm{~g}$ shows a relatively big assembly in which the two clusters are parallel to each other and only slightly out-of-phase. Three extra half layers are marked, which are mainly caused by the changing orientations of the "bridging" layers in the junction. The nonparallel "bridging" layers may be partly caused by the fluctuation of the molecule orientation. The detailed structures in Figs. 4e-4g also suggest that some of the layers are bridged to two of the layers in the neighboring cluster instead of the normal "one-on-one" correspondence. In addition, the local layer spacing near the complex junctions can largely deviate from the average value $(\sim 2.95 \mathrm{~nm})$. For example, we have observed layer spacings smaller than $2.5 \mathrm{~nm}$ or larger than $4 \mathrm{~nm}$. The successful observation of the above detailed structures demonstrates the unique advantage of direct TEM imaging combining high resolution and projection along the whole thickness of a thin specimen.

In Figs. 5a-5e and 6a, we show cryo-TEM textures of the lyotropic chromonic LCs (DSCG and SSY) prepared by the technique of thin film plunge freezing (Fig. 2d). At the quenching temperature (room temperature), the chromonic LC samples exhibit isotropic (6.2\% DSCG) and uniaxial nematic (15\% DSCG and 30\% SSY) phases, respectively, revealed by PLM (not shown). The uniform contrast of the suspended 6.2\% DSCG thin film (Fig. 5a) and the stripes with bright/dark contrast in 15\% DSCG (Fig. 5b) match the isotropic and uniaxial nematic structures, respectively. The dark stripes can be understood as the elongated chromonic aggregates formed by face-to-face packing of the DSCG molecules in water. Fig. 5c shows the corresponding FFT pattern of the nematic texture in Fig. 5b, and Fig. 5d is a magnified image of the marked local area. The length of the columnar aggregates was measured to be $25-80 \mathrm{~nm}$. The values match reasonably well the estimated upper limit of aggregate length, $20 \mathrm{~nm}$, in the isotropic phase of 14\% DSCG immediately above the nematic-to-isotropic phase transition (Nastishin et al., 2004). The spacing between the stripes varies from $3.6 \mathrm{~nm}$ to $5 \mathrm{~nm}$, and averages around $4.2 \mathrm{~nm}$, which matches the measured value from the FFT pattern. The spacing of the aggregates measured from the side-views can be underestimated due to the projection effect. In addition, the aggregates seem to assemble into some bundles of tens of nanometers in size, which rules out any long range order and may partly account for the diffused bright spots in the FFT pattern. The nematic arrangement of the aggregates is also evidenced by the frequent observation of groups of dark 
dots (Fig. 5e), which should be small bundles of aggregates oriented perpendicular to the specimen surface. The relative positions of the aggregates in each bundle are rather random, consistent with the nematic structure. It is expected that in the hexagonal columnar phase, at higher concentration, the aggregates would form a lattice. The distance between neighboring aggregates, measured from such top-views, ranges between $4 \mathrm{~nm}$ and $5 \mathrm{~nm}$ with an average around $\sim 4.6 \mathrm{~nm}$, slightly larger than the measured spacing using the side-view images and matching the XRD measurements closely (Tortora et al., 2010; Collings et al., 2010). The DSCG samples contain a relatively small amount of organic materials, only $6.2 \mathrm{wt} \%$ and $15 \mathrm{wt} \%$. Because of the high water content and low viscosity, the TEM specimen preparation by thin film plunge freezing, can be performed in a manner similar to the preparation of aqueous biological samples.

Fig. 6a shows a cryo-TEM image of the SSY solution, in which the percentage of the organic component is higher, about $30 \mathrm{wt} \%$. With the decrease of the water content, the lyotropic nematic phase becomes rather viscous, making it challenging for the blotting process before the plunge freezing. As a result, prolonged blotting or larger blot force are often required to make a thin specimen, which may cause change in water concentration and modification of the LC structure. Reduced amount of solution $(<0.5 \mu \mathrm{l})$ applied onto the TEM grids, short blot time and small blot force were found to help the preservation of the chromonic structure. In Fig. 6a, the projected distance between the aggregates are normally between $2 \mathrm{~nm}$ and $2.4 \mathrm{~nm}$, which is consistent with the XRD measurement (Park and Lavrentovich, 2012; Park et al., 2011) and the result from the "bulk" approach shown in Fig. 6b. Similar to Fig. 5b, the aggregates in the same hole have very similar orientations, influenced by the hole edges of the supporting carbon film.

\section{Application of cryo-electron microscopy of vitreous section (CEMOVIS) in lyotropic LCs}

In this study, we apply the so-called CEMOVIS to lyotropic LCs, i.e., high pressure freezing is employed to obtain vitrified "bulk" lyotropic LCs, followed by cryo-ultramicrotomy (Fig. 2e) and cryo-TEM. Fig. 6b shows a CEMOVIS image taken from the SSY solution. The chromonic nematic LC has a relatively low water percentage $(65 \%)$, and can be directly high pressure frozen without any cryoprotectant. The successful vitrification is evidenced by the lack of crystalline ice (hexagonal or cubic). Fig. 6b demonstrates that the "bulk" CEMOVIS approach yields very similar results to the "thin film" approach in terms of the aggregate assembly and spacing.

In contrast, the DSCG samples with higher water contents $(93.8 \%$ and $85 \%)$ cannot be directly vitrified by high pressure freezing. In this study, we employed dextran $(20 \%$ dextran for the $6.2 \% \mathrm{DSCG}$, and $10 \%$ for the $15 \% \mathrm{DSCG}$ ), one of the most widely used cryoprotectants, to 
achieve successful vitrification. Isotropic and nematic structures similar to those shown in Fig. 5 have been observed in the two samples, respectively. Figs. $5 \mathrm{f}$ and $5 \mathrm{~g}$ are two CEMOVIS images of the 15\% DSCG with 10\% dextran, showing the side view (Fig. 5f) and top view (Fig. 5g) of the aggregates from predominantly nematic regions.

It should be emphasized that the influence of cryoprotectants such as dextran on the structure of lyotropic LCs is much more complicated compared to the case of biological samples in general. Lyotropic chromonic LCs are very sensitive to concentration, and may experience strong condensing effect and dramatic structure change with additives competing for water as depletion agents, as demonstrated for DSCG and SSY with added polyethylene glycol (Tortora et al, 2010; Park et al, 2011) and for LC phases of DNA oligomers (Zanchetta et al., 2008), cellulose (Edgar and Gray, 2002), and viruses (Dogic et al., 2004) in presence of dextran. In a careful study on the influence of dextran on the DSCG phase diagram, we used temperature-dependent PLM to observe the phase transitions in four solutions, including 6.2\% DSCG with $20 \%$ dextran, and three $15 \%$ DSCG solutions with $10 \%, 15 \%$, and $20 \%$ dextran, respectively. The addition of dextran did not change the isotropic phase of the $6.2 \%$ DSCG at room temperature, but significantly enhanced phase separation in the 15\% DSCG solution. At room temperature, only biphasic states (nematic and isotropic phases for the 10\% dextran solution, and hexagonal and isotropic phases for the $15 \%$ and $20 \%$ dextran solution) were observed in the $15 \%$ DSCG sample, while nematic single phases only existed at much lower temperatures. PLM measurement has been demonstrated to be critical for the LC solutions involving cryoprotectants in order to make sure that the desired phase is studied by the CEMOVIS technique. Systematic efforts are still needed to understand the influences of different cryoprotectants as well as the high pressure freezing process (Richter, 1994). It should be also mentioned that our comparative studies using both thin film (plunge-freezing) and bulk (CEMOVIS) approaches on the same samples $(6.2 \%$ DSCG $+20 \%$ dextran, $15 \%$ DSCG $+10 \%$ dextran, and 15\% DSCG $+20 \%$ dextran) yield very similar results. The consistent outcomes from the two very different approaches further demonstrate the validity of the techniques.

Despite the complication of cryoprotectants in high water percentage samples, the benefits of the "bulk" approach are obvious. The CEMOVIS technique effectively avoids the confinements imposed by the surfaces of the thin liquid film and the hole edges of the carbon layer on the TEM grids, thus the observed structure is more representative of the bulk materials. For example, in thin film approach, the orientation of the aggregates tends to be parallel to the liquid surface and is greatly affected by the hole shape, and the perpendicular bundles can only be observed at the edge of the holes and mostly consist of small numbers of aggregates. While in the bulk approach, different aggregate orientations can be more equally seen, and the bundle size is often a few hundreds of nanometers or even larger (Fig. 5g). Besides, the local orientation of an 
aggregate bundle observed in bulk approach often varies slightly from the nearby ones, and nearly $90^{\circ}$ variation has been observed in the SYY sample. In addition to the technical challenges involved, a major disadvantage of the bulk approach is the artifacts (e.g., knife marks, crevasses, and compression) which can be introduced during cryo-sectioning (Studer et al., 2008; Han et al., 2008). In contrast, the plunge freezing of thin films usually results in cleaner TEM specimen thus TEM images without noticeable artifacts. In Fig. 2f, FFTEM is also shown as an alternative to the CEMOVIS after the high pressure freezing process. It should be mentioned that our initial attempt of FFTEM and cryo-SEM (not shown) on the 15\% DSCG sample failed to yield columnar aggregate-related structures, probably due to the facts that the fracture surface did not correlate closely with the aggregates and/or the replication process did not provide sufficient resolution.

Considering the overall similarity of the results generated, we believe that thin film and bulk approaches can both work for a wide range of lyotropic LCs if used properly. To avoid the complications of cryoprotectants, thin film plunge freezing is preferred for samples with high percentage of water. While for samples with less water, the bulk CEMOVIS approach using high pressure freezing and cryo-ultramicrotomy is more favored to get reliable results. It should be also mentioned that for very viscous lyotropic LCs, thin film plunge freezing and "bulk" high pressure freezing may both encounter great technical challenges. An ongoing effort is being made for such highly viscous lyotropic materials.

\section{Combination of direct cryo-TEM imaging and indirect FFTEM}

As described earlier, LC materials are probably one of the most complicated samples for TEM studies. There is basically no one-for-many routine at the current stage. The available techniques, often developed initially for bio-materials, all have obvious advantages and disadvantages when applied to LCs, as summarized briefly later in the paper. On the other hand, the different techniques are complimentary, and their combination can provide comprehensive characterization for many LCs. In this study, we performed cryo-TEM using both thin film and cryo-sectioning, and FFTEM on thermotropic CB7CB and lyotropic DSCG. Here we present results from complimentary FFTEM and cryo-TEM revealing the nanoscale structure of the peculiar nematic phase, the so-called twist-bend nematic $\left(\mathrm{N}_{\mathrm{tb}}\right)$ in CB7CB with molecules of a dimer type.

As Borshch, et al. (2013) summarized, there can be three types of nematic structures. Besides uniaxial nematics $(\mathrm{N})$ introduced earlier, another well-known mesophase is the chiral (cholesteric) nematic phase $\left(\mathrm{N}^{*}\right)$ in which the director forms a right-angle helix, twisting in space around the helical axis and remaining perpendicular to this axis. While in the strikingly different structure $\mathrm{N}_{\mathrm{tb}}$ as predicted by Meyer (1976), Dozov (2001), and others (Memmer, 2002; Shamid et al., 
2013), the director forms an oblique helicoid, being tilted (rather than perpendicular) to the axis of twist. Fig. 7a shows schematics of dimeric molecules forming a single spiral (left-handed) and filling a small space in the proposed $\mathrm{N}_{\mathrm{tb}}$ structure. Note that in both $\mathrm{N}^{*}$ and $\mathrm{N}_{\mathrm{tb}}$, director twist does not imply modulated density of the material (in contrast to the case of smectics, in which the layered structure is associated with the density modulation). Thus, the 1D periodic structure along the helix axis is defined only as a periodic change in local molecular orientation not associated with any detectable modulation in mass or electron density (Panov et al., 2010; Cestari et al., 2011; Chen et al., 2013, Borshch et al., 2013). The most striking feature of $\mathrm{N}_{\mathrm{tb}}$ helical structure is that its period is very small, on the order of $10 \mathrm{~nm}$, which is by two orders of magnitude smaller than the pitch of the chiral nematics formed in materials such as cholesterol derivatives. As a result, the nanoscale periodicity of the $\mathrm{N}_{\mathrm{tb}}$ phase has been established only very recently, in 2013, thanks to TEM experiments (Chen et al., 2013; Borshch et al., 2013). Moreover, the TEM experiments allowed Borshch et al. (2013) to establish that the structural organization follows the geometry of the oblique helicoid rather than, for example, a mixed splay-bend state, also predicted theoretically (Meyer, 1976; Dozov, 2001; Memmer, 2002; Shamid et al., 2013).

To demonstrate such a 1D periodic structure, the surface sensitive FFTEM turned out to be essentially useful. Though the low temperature fracture can occur along various orientations, it is easier to fracture along the length of the molecules since less energy/area is required to break side-side bonds (Berreman et al., 1986). In CB7CB, the favored fracture planes are those parallel to the helix axis, so it is possible to visualize the 1D periodic structure if smooth fracture and proper shadowing can both be achieved. In practice, such desired fracture and replication do present and well-defined periodic layers can be observed (Fig. 7b). The layer spacing is considered to be corresponding to the pitch of the helix structure (Chen et al., 2013; Borshch et al., 2013). Fig. 7b shows clearly domains of several hundreds of nanometers to microns in size. Most layers are either very straight or slightly curved (the inset of Fig. 7b). Several expected fracture configurations have been observed. If the fracture occurs almost perfectly parallel to the helix axis (parallel fracture), clean layer structure can be revealed and the pitch can be measured accurately (domain I in Fig. 7b and Fig. 7c). If the fracture surface has a tilting angle relative to the helix axis (oblique fracture), steps can be formed with the terraces still showing basically the same features as the parallel fracture (domain II in Fig. 7b).

As an alternative to the steps in oblique fracture, Bouligand arches (Bouligand et al., 1968) can be formed for helix structures such as the $\mathrm{N}_{\mathrm{tb}}$ phase, as shown in Figs. $7 \mathrm{~d}$ and $7 \mathrm{e}$ corresponding to different fracture angles. The arch structure observed in $\mathrm{CB} 7 \mathrm{CB}$ is very different from the arch structure observed in chiral nematics, as discussed in details by Borshch et al., (2013). In the chiral nematic phase, each $180^{\circ}$ twist of the director results in a complete arch, in which the 
director imprint rotates (through in-plane bend and splay) by $180^{\circ}$. In contrast, the oblique helicoidal structure of the $\mathrm{N}_{\mathrm{tb}}$ phase results in two other types of Bouligand arches: (i) an alternating system of "wide" and "narrow" arches (Fig.7d), each of which corresponds to a $180^{\circ}$ twist, and (ii) a periodic system of incomplete arches, in which the imprint does not reorient by $180^{\circ}$ (Fig.7e). Neither of the two latter types of the Bouligand arches is characteristic of a chiral nematic. Note that the wide and narrow arches in Fig.7d have a different affinity to fracturing and subsequent metal deposits, thus they might show a different contrast in the resulting FFTEM textures of replicas.

The above results indicate clearly a $1 \mathrm{D}$ periodic structure at the fracture surface and a helix axis in the bulk material. As Chen et al., (2013) reported, the layer contrast in CB7CB is considerably weaker compared to other layered LC systems with mass and electron density modulation. Similar absence of density modulation was reported by Borshch et al (2013) for another $\mathrm{N}_{\mathrm{tb}}$ material. For parallel fracture, the period in most of the domains ranges between 8.0 and $8.2 \mathrm{~nm}$. In Fig. $7 b$, the pitch was measured to be $8.05 \mathrm{~nm}$ from the image and the FFT patterns. In addition to the domains with narrow pitch distribution, larger pitches can also be found (e.g., 8.6 $\mathrm{nm}$ ), which can be partly attributed to the cosine relation between the layer spacing in a parallel fracture and the measured spacing in oblique fracture. An interesting finding is that some domains show smaller pitches especially in the peripheral regions, in contrast to the uniform pitch found in most of the individual domains (e.g., Fig. 7b). Figs. $7 \mathrm{f}$ and $7 \mathrm{~g}$ show two such domains, where the pitches near the center of the domains are $\sim 7.6 \mathrm{~nm}$, only slightly smaller than the normal ones, but the measured periods near the boundaries are $<3 \mathrm{~nm}$. In Fig. $7 \mathrm{~g}$, the layer spacing gradually increases from $<3 \mathrm{~nm}$ near the boundary to $7.6 \mathrm{~nm}$ near the center of the domain with a one-on-one correspondence, which rules out the possibility of the half-pitch situation (Ihn et al., 1992). In addition, in both cases, the layer orientations vary to accommodate the boundaries. The above results may indicate the flexible nature of the $\mathrm{N}_{\mathrm{tb}}$ phase, which may adopt different conformational states with small energy difference depending on the local environment. It is also worth mentioning that some detailed features of $\sim 1 \mathrm{~nm}$ in size and distance are resolved near the boundaries, though rather poorly, suggesting the potential use of FFTEM in high resolution imaging in some special LC systems.

The free surface of $\mathrm{CB} 7 \mathrm{CB}$ sets the director orientation perpendicular to itself (the so-called homeotropic alignment), which makes suspended thin films suitable to study the top-view structure. Figure 8a is a typical cryo-TEM image of suspended CB7CB thin films quenched at $95^{\circ} \mathrm{C}$, showing clearly column-like structure and narrow low-density areas between some of the columns. The columns tend to have more or less circular shape and have an average size of $\sim 27$ $\mathrm{nm}$ measured over a large area. Our results also show that the column structure from the suspended films can be influenced by thermal treatment and the film thickness. Careful 
examination of the FFTEM images reveals the side-view of the column-like texture in some local regions. For example, in Fig. 7b, we use arrows to denote the column boundaries from the top of the layered domain, emphasizing the distinct column shape near the domain boundaries. The column boundaries can be seen as narrow areas often with a discontinuity of the layered structure and elongating roughly along the layer normal in the image, which is also revealed by the diffuse scattering perpendicular to the ordering direction in the FFT pattern. In the image, the 8 marked adjacent column boundaries have an average distance of $\sim 26 \mathrm{~nm}$. We have carefully examined the layer structure across the column boundaries in the FFTEM images. Along with a few exceptions, most of the layers exhibit no shift along the layer normal, which may mean that the helix structure tends to stay "in phase" for neighboring columns. The inset of Fig. 7c is a lowpass filtered gradient image of the marked rectangular area, which enhances the layer characteristics and confirms the "in phase" configuration. The top-view of the columns can also be identified in the FFTEM images. The inset in Fig. 8a is a typical local region with a cluster of circular columns, corresponding to a fracture perpendicular to the helix axis. It should be pointed out that it is difficult to correlate such features in FFTEM to columns without the cryo-TEM results. Except those layer-related features, the FFTEM images tend to be complicated at the presence of artifacts and various features related to the fracture morphology.

Despite of the consistency discussed above, the origin of the column structure cannot be determined unambiguously at this stage. The columns do not appear to be as common in the FFTEM images as in the thin films if we assume that the subtle feature can be well replicated in the specimen preparation process. In addition, such "leopard" pattern can sometimes be found in plunge-frozen cryo-TEM specimens with water-based buffer due to the local concentration variation (e.g., loss of water). Though the same cause does not apply to the single-component $\mathrm{CB} 7 \mathrm{CB}$, the column structure may still be an unusual feature in the bulk material but enhanced greatly in thin films.

Our current results suggest that the column structure with their long direction along the helix axis may be introduced by the coexistence of right- and left-handed $\mathrm{N}_{\mathrm{tb}}$ columns, while very small amount of residual high temperature $\mathrm{N}$ phase may exist at the column boundaries (Borshch et al., 2013). A strong evidence is that upon doping with chiral dopant (1-1.5\% right-handed limonene), the column size increases dramatically (Fig. 8b) under the same thermal processing condition, as the chiral dopant keeps more columns in the same handedness. The columns in the doped films often have irregular shapes. In Fig. 8b, a column with typical shape is highlighted, which shows that the smallest dimension of the columns is very similar to that found in the pure films, but a few nearby columns may merge together due to the same chirality. To compare the column size, we simply compare the domain numbers in regions with the same area for both pure and doped films. For example, for a $200 \mathrm{~nm} \times 200 \mathrm{~nm}$ area, 50-60 columns can be found in pure films, 
while only 20 or even less in doped films. Recently, Hoffmann et al. (2014), proposed a similar model based on nuclear magnetic resonance (NMR) measurement of a similar LC compound, wherein the molecules organize into highly correlated microscopic domains of opposite chirality.

Another possibility of the formation of the column structure observed in thin films is due to the confinement of the thin films. As the recent simulation (Fukuda and Žumer, 2011) showed, twodimensional Skyrmion lattice embedded in topographic defects can develop in a highly chiral nematic LC thin film with surfaces favoring homeotropic alignment. The two possibilities discussed above both indicate a high chirality structure, consistent with the proposed twist-bend nematic structure. In addition, our initial cryo-TEM result (not shown) using "bulk" cryosectioning routine (Fig. 2b) did not reveal layered structure consistent with the FFTEM result, which may be considered as a further evidence that there are no modulation of electron/mass density for the $\mathrm{N}_{\mathrm{tb}}$ phase. The above comprehensive study combining FFTEM and cryo-TEM clearly shows that the low temperature nematic phase of $\mathrm{CB} 7 \mathrm{CB}$ has $1 \mathrm{D}$ periodic helix structure and match the predicted $\mathrm{N}_{\mathrm{tb}}$. The successful revealing of the $1 \mathrm{D}$ periodic structure is attributed directly to the replication of the smoothly fractured surface in FFTEM.

\section{Surface/thickness effect, cooling rate and radiation damage}

The routines summarized in Fig. 2, including cryo-TEM and FFTEM, all aim at taking snapshots of microstructures of LC materials. To be successful, several critical requirements have to be satisfied for both "thin film" and "bulk" approaches: 1) the pre-freezing samples should have the desired native structure; 2) the samples have to be quenched effectively at a sufficient cooling rate to prevent from any further phase transition and structure change during and after the freezing process; 3) any possible misleading structure deformation or change introduced during the specimen processing after the quenching should be kept minimum. For thin film approach, the main concern is to make thin films with representative structure. The limited thickness may lead to undesired surface/interface effect. While for the "bulk" approach, it is often challenging to achieve sufficient cooling rate to quench the structure effectively. Damage and deformation can also be introduced during the processing of the "bulk" material into TEM specimens. In addition, direct TEM imaging routines all suffer from radiation damage, which may become a serious issue for those highly sensitive materials.

As already indicated, the surface that LC makes contact with can often strongly influence the molecular orientation near LC surface, i.e., the so-called surface anchoring, which can be simply understood as epitaxial growth of solids on substrates (Jérôme, 1991). Depending on the substrate surface configuration, the LC molecules can be perpendicular (homeotropic), parallel (planar) or tilted relative to the substrate surface. If the LC film is too thin, the internal structure can be dominantly governed by the surface and does not represent the bulk structure which is 
often desired in TEM studies. Obviously, the surface effect has profound influences on the TEM results using thin film approach and deserve more detailed studies. Here we present some of our results to demonstrate the effect.

For the thin film approach used here, there exist two LC/air interfaces for a suspended LC film (if ignoring the influence of the hole edges in the supporting carbon film), while one LC/air interface and one LC/carbon interface for supported thin film. Simple treatment of the carbon film can affect the LC structure greatly. Figs. 9a and 9b compare the CB7CB thin film deposited on plasma treated and untreated thin carbon grids. The plasma-treated carbon exhibits much better wetting property to the $\mathrm{CB} 7 \mathrm{CB}$, resulting in large area of relatively uniform thin film. Similar to the suspended films (Fig. 8a), the CB7CB forms a column structure in the whole film (Fig. 9a). The upper inset of Fig. 9a shows a magnified image of a local area, showing clearly sharp bright lines in the elongated columns and bright dots in relatively circular columns, respectively. The lines are roughly along the orientation of the long direction of the domains. Such bright lines/dots are not observed in suspended films (Fig. 8), and are considered as some low density topographic defects formed due to the different molecule alignments at the two surfaces. The lower inset of Fig. 9a is a plausible model showing the side-view of a toroidal focal conic domain. The arrows represent the helicoid axes with the same handedness in the domain, which are tangential to the $\mathrm{CB} 7 \mathrm{CB} /$ carbon interface, but perpendicular to the free surface. Note that the helical axes are more or less straight due to the periodic twist-bend structure. AA is a defect line in an axially-symmetric circular column, and a narrow defect wall (with its long direction along the projection direction) in an elongated column, corresponding to the bright dots and lines in Fig. 9a, respectively. On the other hand, on the untreated film, the LC material typically tends to form featureless droplets with increasing thickness from the edge to the center. The columns of $\sim 20-40 \mathrm{~nm}$ in dimension can only be seen in thick droplets and become a less pronounced feature as the thickness increases further, as seen in Fig. 9b. Bright lines/dots can still be identified at the column centers. The above result may indicate that the carbon film (treated or untreated) favors director orientations other than the homeotropic configuration for CB7CB, so the influence of the interface with air can only be seen when the material is far enough from the carbon film. On the other hand, the current result may also suggest that the column structure is only stable in certain thickness range, which should be able to be clarified with more quantitative structure-thickness correlations.

The strong surface effect in LCs raises doubts on the general use of the thin film approach and make it often necessary to discuss the validity of the observed results. In practice, this can be done by comparing results in films with different thicknesses by depositing different amount of LC solution on the carbon films. Even more conveniently, wedge-shaped areas with gradually changing thickness can be found frequently. For example, Fig. 9c shows an image of the 3RBC- 
$\mathrm{N}$ thin film with smectic clusters. The intensity profile can be considered as a rough indication of the varying thickness, which can be further correlated to thickness through EELS or thickness mapping using energy filtered TEM. No smectic layers can be found in the very thin region, ruling out the possibility that the formation of the smectic clusters are due to surface effect (Ruths et al., 1996). Instead, the surface effect at least prohibits the formation of the smectic clusters with layers perpendicular to the surface. It can also be seen that the layered clusters can still be resolved in the very thick part of the sample (the inset of Fig. 9c). EELS measurement showed that the thick part should be at least 4 times of the inelastic mean free path, i.e., thicker than $500 \mathrm{~nm}$. The layer length and layer number in the thick area are basically the same as those found in thinner regions (100-200 nm). Clusters with very few layers (e.g., 2) can also be seen in the thick parts of the specimen. Above results show that the observed smectic clusters stay the same for a wide range of thickness, which validate the application of the thin film approach in this material.

Interestingly, in some of the thin areas, very narrow smectic clusters can be found (Fig. 9e). The layer width can be even shorter than $3 \mathrm{~nm}$, while the layer number within the clusters varies from 1 to 9 , which is comparable to those in the normal clusters. The layer spacing is considerably larger $(3.1 \mathrm{~nm}-3.5 \mathrm{~nm})$ compared to the wider clusters. Because the confinement is in the thickness direction, it may be reasonable to assume a similar cluster depth in the thickness direction. Considering that the interface prevents the layered structure formation, the short layers smectic clusters may indicate the rather strong tendency of the $3 \mathrm{RBC}-\mathrm{N}$ molecules to form layered structure as the surface impact becomes weaker.

Carbon films also play an important role in the observed structure of suspended chromonic lyotropic LC thin films prepared by plunge freezing (Figs. 5a-5d and 6a). As we mentioned earlier, most columnar aggregates tend to orient along the long direction of the holes, and probably prefer to be parallel to the liquid surface. Inside each hole, the aggregates have basically the same orientation except that the shape of the hole affects those aggregates near the edge. This can be seen more clearly in the region with smaller holes, as shown in Fig. 9f. The areas near hole edges are under the competing influences of the liquid surface and the carbon edge. As a result other than lying along the edge and parallel to the liquid surface, small aggregate bundles perpendicular to the liquid surface can be found near the hole edge (Fig. 9f).

In this study, we only employ LC surfaces making contact with air and carbon films. In principle, a variety of materials and surface treatments can be used. In addition, the surface/interface impact may provide a way to modify the LC structure or orientations using the thin film approach or simulate the working condition of LC devices in TEM studies. 
In general, it is more difficult to quench samples with their internal structure intact for bulk samples than thin films since the cooling rate inside the sample is mainly determined by the heat conductivity of the material, and more difficult for lyotropic LCs than thermotropic LCs due to the high percentage of water which has unusually high heat capacity. For example, to vitrify a water based sample requires a cooling rate of the order of $10^{5} \mathrm{~K} / \mathrm{s}$. In practice, it is straightforward to use liquid ethane as cryogen for both thermotropic and lyotropic LC thin films. Thermotropic LC thin films normally require a less demanding cooling rate due to considerably slower molecular diffusion and smaller heat capacity. As a result, the much more convenient quenching in liquid nitrogen often gives the same results as liquid ethane, revealed by comparing the results using liquid nitrogen and liquid ethane as cryogens (not shown).

Generally speaking, plunge freezing of bulk materials may only work for samples of a few microns in thickness. However thinner samples may experience increased surface effects and also make it challenging for the follow-on processing (e.g., cryo-sectioning and freezing fracture). The application of high pressure freezing initiated in this study seems to be a reliable method for bulk lyotropic LCs up to a few hundreds of microns in the smallest dimension. More efforts are still needed to improve the structure preservation in high water percentage samples, clarify the influence of the cryoprotectant, and reduce the damages in the following cryosectioning process. For thermotropic LCs, currently there is no equivalent technique to high pressure freezing for lyotropic LCs to allow structure quenching at a lower cooling rate. Fortunately, for some of the thermotropic LC materials, e.g., CB7CB (Chen et al., 2013), transitions can be supercooled, which helps to preserve the native structure even at a slow cooling rate. But for other materials without supercooling, extra caution has to be taken. In practice, different structure indicating different cooling rate can be observed at different depth using the combination of plunge freezing and cryo-ultramicrotomy (not shown).

Direct cryo-TEM imaging techniques used here all suffer from radiation damage to the electrontransparent specimens. Our results show that the radiation damage of the LC thin films first exhibits as the dissipation of the ordered structure (smectic layers in this study) due to the very weak intermolecular force stabilizing the ordering. Such subtle damage can often lead to the overlooking of the ordered structures present, as it does not involve observable structure and shape changes at a large scale. In some materials, the damage to the ordering may happen at very low doses (even $<2 \mathrm{e}^{-} / \AA^{2}$ ), making it difficult to capture an image with reasonable $\mathrm{S} / \mathrm{N}$ ratio. On the other hand, those mass loss related damages, leading to larger scale structure changes, occur at much higher dose. As a result, atomic resolution imaging can often be obtained before any obvious shape changes, provided ordered structures are not involved or not the target of the investigation. For example, Fig. 10a shows a lattice image of gold nanoparticles doped in 5CB. The 5CB thin film also exhibits sufficient stability for spot-mode STEM imaging and 
spectroscopy (e.g., EELS and EDS) measurement. One benefit of STEM is to increase the visibility of some features in thick regions owing to the emphasis of atomic number difference and the minimized influence of crystal orientation on the contrast. Fig. 10b shows a bright field TEM image of gold nanoparticles dispersed in a thick region of 5CB. The small nanoparticles can be hardly identified. In contrast, a STEM Z-contrast image of a similar area (Fig. 10c) shows an abrupt contrast even for nanoparticles of $\sim 1 \mathrm{~nm}$ in diameter. Fig. 10d presents the corresponding EDS spectra taken by scanning a nanometer-sized box over the top of an individual nanoparticle (Gao et al., 2003a) and the surrounding 5CB. As organic materials, the radiation damage of LC materials basically fits into the description given by Egerton, et al. (2004). Several ways can be considered to minimize the damage: lowering the dose combined with a more sensitive camera, use of a higher beam energy and of a thicker specimen. In practice, field emission electron source often causes more irradiation damage. A traditional $\mathrm{LaB}_{6}$ source can reduce the damage yet still keep a sufficient resolution for LC studies (Egerton et al., 2004).

\section{A brief comparison of the different specimen preparation techniques for LCs}

In this study, we aim to establish an easily accessible toolset for the TEM specimen preparation of a wide range of LCs. Based on the earlier discussion, a brief comparison of the different techniques is given in Table 1. At the current stage, there is simply no one-for-many routine. Each method can be powerful for certain materials, but can also be difficult to apply to others. For example, direct cryo-TEM allows high resolution imaging and the application of a variety of TEM techniques, but suffers from radiation damage; while the replica FFTEM provides basically the opposite advantages and disadvantages. In addition to the routines mentioned above, several other techniques, often originally developed for biological samples, can also be applied to LC materials. For example, thermotropic thin films can also be obtained by spin coating or suspension on water (Voigt-Martin and Durst, 1987), which may result in uniform thickness. Jet freezing (Severs, 2007) and slam freezing (Mondain-Monval, 2005) can be used as alternatives in freezing process and may provide more or less higher cooling rate for some special LCs than plunge freezing. Slam freezing with liquid helium cooled cooper block has been shown to provide better structure preservation in FFTEM of some LC materials (Leforestier et al., 1996). A self-pressurized rapid freezing was proposed using only plunge freezing and sealed capillary tubes (Leunissen and Yi, 2009), which may be able to be applied to some lyotropic LCs as a lowcost version of combined high pressure freezing and cryo-sectioning (Fig. 2e). Another interesting specimen preparation technique uses similar procedure to the plunge freezing FFTEM (Fig. 2f): a holey carbon coated TEM grid is placed between the two planchettes during the freezing process and some thin areas may remain on the film upon fracture, which can be used for direct cryo-TEM observation (Belkoura et al., 2004). 
The very convenient-to-use thin film approach allows the highest cooling rate and avoids further specimen processing after the freezing, which usually results in the best imaging quality and preservation of the pre-freezing structure. But surface effect has to be considered especially for structures with dimension comparable to the thickness. Another practical advantage of thin film approach is that very small amount of material is required compared to bulk approach, which is often important for some thermotropic LCs due to possible complicated synthesis process.

FFTEM and cryo-sectioning, both using bulk approach to minimize surface effect, require effective quenching of thick samples, and often show similar results (not shown) especially for thermotropic LCs due to similar freezing process. As a widely used technique, FFTEM effectively avoids radiation damage and uses convenient room temperature observation, though the resolution is limited to a few nanometers. We also demonstrate the critical role of the surface sensitivity of FFTEM in this study, which may make FFTEM an irreplaceable technique for LCs at the current stage. Artifacts can be common in FFTEM images and extra caution needs to be taken to explain the results because the replica shadowing/contrast totally depends on the fracture surface morphology and deposition direction, and does not necessarily correlates with the intrinsic structure in LC materials. The freezing of thick sample raises concerns of insufficient cooling rate to preserve the intrinsic structures except for those thermotropic LCs with supercooling property. Plunge-freezing of thick lyotropic LCs leads to water crystallization, and modification of local structures. (Mondain-Monval, 2005) But FFTEM of lyotropic LCs can still work for low-resolution imaging which is the focus of most FFTEM studies.

Cryo-ultramicrotomy seems to combine the advantages of both thin film technique and FFTEM, but some of the disadvantages as well. Compared to FFTEM, cryo-sectioning allows high resolution direct imaging, and the process-induced artifacts (e.g., knife marks, crevasses, and compression) are relatively easier to identify. The CEMOVIS technique, combining high pressure freezing, cryo-sectioning and cryo-TEM, allows the observation of lyotropic LCs beyond the resolution of FFTEM. In contrast to the little control of fracture process in FFTEM, cryo-sectioning makes it possible to look at different depth of the sample. As an alternative to cryo-sectioning, focused ion beam (FIB) (Strunk et al., 2012) may turn out to be very useful especially for some challenging LC materials (e.g., high viscosity lyotropic LCs). The advantages of cryo-FIB include less mechanical deformation (knife marks, crevasses, and compression), more convenient preparation of specimen in different orientations, and higher efficiency in using the regions frozen at fast cooling rate.

In conclusion, we present our on-going efforts towards high resolution direct TEM observation of thermotropic and lyotropic LCs. A series of specimen preparation techniques have been summarized and tested to provide a comprehensive toolbox for TEM studies of LC materials. The complications in these techniques are also discussed. We demonstrated that the current 
available specimen preparation and low-dose cryo-TEM techniques allow sub-nanometer resolution direct TEM imaging of a wide range of LCs, which can become part of the solution to the lack of effective structure probe at the molecular level for LC studies. We also demonstrate that the currently more widely used replica TEM technique, FFTEM, is complementary to direct cryo-TEM observation. Their combination is highly recommended to get comprehensive information on the LC materials, which can be especially important at the current stage when there is no obvious advantageous approach.

The direct observation of LCs opens ways to apply a variety of TEM techniques beyond lowdose imaging to these fascinating but challenging materials, for example, diffraction (Gao et al., 2003b; Zuo et al., 2003; Zuo et al., 2011), tomography, STEM, and spectroscopy. Maybe more importantly, direct observation beyond taking snapshots using cryo-TEM may lead to the possibility of in situ observation of the dynamic processes using in situ TEM. The current development in high sensitivity electron-counting camera (Li et al., 2013), in situ liquid cell technique (Zhu et al., 2013), and ultrafast TEM (Kim et al., 2008) make it promising to look at LC materials with temporal resolution in the near future.

Acknowledgement:

The TEM results shown in this paper were taken at the TEM Lab of the Liquid Crystal Institute (LCI) at Kent State University, supported by the Ohio Research Scholars Program Research Cluster on Surfaces in Advanced Materials. MG thanks the LCI Characterization Facility for the support of his research. ODL research is supported by NSF grants DMR 1104850 and 1121288. AK and MGT were supported by the EU project BIND (contract 216025). LF acknowledges the National Science Foundation of China (Grant No. 61172011). The authors thank Dr. Kim Rensing of Leica for the FFTEM and cryo-SEM of high pressure frozen DSCG. MG thanks the following people for the very helpful discussion: Mr. Michael Boykin from Mager Scientific, Inc., Prof. James Gleeson, Prof. Samuel Sprunt, Dr. Yannian Li, Dr. Chenming Xue, and Mr. Andrew Konya from Kent State University; Prof. Haixin Sui from Wadsworth Center, and Dr. Xing Zhang from University of California at Los Angeles. 


\section{References:}

Belkoura L, Stubenrauch C, and Strey R. 2004. Freeze fracture direct imaging: a new freeze fracture method for specimen preparation in cryo-transmission electron microscopy. Langmuir 20: 4391-4399.

Berreman DW, Meiboom S, Zasadzinski JA, Sammon MJ. 1986. Theory and simulation of freeze-fracture in cholesteric liquid crystals. Phys Rev Lett 57: 1737-1740.

Borshch V, Kim Y-K, Xiang J, Gao M, Jákli A, Panov VP, Vij JK, Imrie CT, Tamba MG, Mehl GH, Lavrentovich OD. 2013. Nematic twist-bend phase with nanoscale modulation of molecular orientation. Nature Comm 4: 2635.

Bouligand Y, Soyer, MO, Puiseux-Dao S. 1968. La structure fibrillaire et l'orientation des chromosomes chez les Dinoflagellés. Chromosoma 24: 251-287.

Bunning TJ, Vezie DL, Lloyd PF, Haaland PD, Thomas EL, Adams WW. 1994. Cholesteric liquid crystals: image contrast in the TEM. Liq Cryst 16: 769-781.

Cestari M, Diez-Berart S, Dunmur DA, Ferrarini A, de la Fuente MR, Jackson DJ, Lopez DO, Luckhurst GR, Perez-Jubindo MA, Richardson RM, Salud J, Timimi BA, Zimmermann H. 2011. Phase behavior and properties of the liquid-crystal dimer 1',7'-bis(r-cyanobiphenyl-4'yl)heptane: a twist-bend nematic liquid crystal. Phys Rev E 84: 031704.

Chen D, Heberling MS, Nakata M, Hough LE, Maclennan JE, Glaser MA, Korblova E, Walba DM, Watanabe J, Clark NA. 2012. Structure of the B4 liquid crystal phase near a glass surface. Chem Phys Chem 13: 155-159.

Chen D, Porada JH, Hooper JB, Klittnick A, Shen Y, Tuchband MR, Korblova E, Bedrov, Walba DM, Glaser MA, Maclennan JE, Clark NA. 2013. Chiral heliconical ground state of nanoscale pitch in a nematic liquid crystal of achiral molecular dimers. Proc Natl Acad Sci 110: 15931-15936.

Costello MJ, Meiboom S, Sammon M. 1984. Electron microscopy of a cholesteric liquid crystal and its blue phase. Phys Rev A 29: 2957-2959.

Collings PJ. 2002. Liquid crystals nature's delicate phase of matter ( $2^{\text {nd }}$ edition). Princeton and Oxford: Princeton University Press.

Collings PJ, Dickinson AJ, Smith EC. 2010. Molecular aggregation and chromonic liquid crystals. Liq Cryst 37: 701-710. 
De Gennes PG, Prost J. 1995. The Physics of Liquid Crystals. New York: Oxford University Press.

Dogic Z, Purdy KR, Grelet E, Adams M, Fraden S. 2004. Isotropic-nematic phase transition in suspensions of filamentous virus and the neutral polymer Dextran. Phys Rev E 69: 051702.

Dozov I. 2001. On the spontaneous symmetry breaking in the mesophases of achiral bananashaped molecules. Europhys Lett 56: 247-253.

Edgar CD, Gray DG. 2002. Influence of dextran on the phase behavior of suspensions of cellulose nanocrystals. Macromolecules 35: 7400-7406.

Egerton RF, Li P, Malac M. 2004. Radiation damage in the TEM and SEM. Micron 35: 399-409.

Egerton, RF. 2011. Electron energy-loss spectroscopy in the electron microscope (Third edition). New York: Springer.

Frommer J. 1992. Scanning tunneling microscopy and atomic force microscopy in organic chemistry. Angew Chem Int Ed Engl 31: 1298-1328.

Fukuda J, Žumer S. 2011. Quasi two-dimensional Skyrmion lattices in a chiral nematic liquid crystals. Nature Communications 2: 246.

Gai PL, Boyes ED. 2009. Advances in atomic resolution in situ environmental transmission electron microscopy and $1 \AA$ aberration corrected in situ electron microscopy. Microsc Res Tech 72: $153-164$.

Gao M, Zuo JM, Twesten RD, Petrov I, Nagahara LA, Zhang R. 2003a. Structure determination of individual single-wall carbon nanotubes by nanoarea electron diffraction. Appl Phys Lett 82: 2703-2705.

Gao M, Scheu C, Tchernychova E, Rühle M. 2003b. Successful application of spatial difference technique to electron energy-loss spectroscopy studies of Mo/SrTiO interface. J Microsc 210: 94-101.

Gilli JM, Kamayé M, Sixou P. 1991. Quenched blue phase, below the glass transition of a side chain polysiloxane: electron microscope studies. Mol Cryst Liq Cryst 199: 79-86.

Han HM, Zuber B, Dubochet. 2008. Compression and crevasses in vitreous sections under different cutting conditions. J Micros 230: 167-171. 
Hara H, Satoh T, Toya T, Iida S, Orii S, Watanabe J. 1988. Cholesteric liquid crystalline polyesters. 1. Cholesteric liquid crystalline copolyesters based on poly(chloro-1,4-phenylene trans-1,4-cyclohexanedicarboxylate). Macromolecules 21: 14-19.

Hoffmann A, Vanakaras AG, Kohlmeier A, Mehl GH, Photinos DJ. 2014. On the structure of the Nx phase of symmetric dimers. Arxiv: 1401.5445.

Hong SH, Verduzco R. Williams JC, Twieg RJ, DiMasi E, Pindak R, Jákli A, Gleeson JT, Sprunt S. 2010. Short-range smectic order in bent-core nematic liquid crystals. Soft Matter 6: 4819-4827.

Hough LE, Jung HT, Krüerke D, Heberling MS, Nakata M, Jones CD, Chen D, Link DR, Zasadzinski J, Heppke G, Rabe, JP, Stocker W, Körblova E, Walba DM, Glaser MA, Clark NA. 2009. Helical nanofilament phases. Science 325: 456-460.

Ihn KJ, Zasadzinski JAN, Pindak R, Slaney AJ, Goodby J. 1992. Observation of the liquidcrystal analog of the abrikosov phase. Science 258: 275-278.

Jérôme B. 1991. Surface effects and anchoring in liquid crystals. Rep Prog Phys 54: 391-451.

Kim JS, LaGrange T, Reed BW, Taheri ML, Armstrong MR, King WE, Browning ND, Campbell GH. 2008. Imaging of transient structures using nanosecond in situ TEM. Science 321: 1472-1475.

Kim YK, Shiyanovskii SV, Lavrentovich OD. 2013. Morphogenesis of defects and tactoids during isotropic-nematic phase transition in self-assembled lyotropic chromonic liquid crystals. J Phys Condens Matt 25: 404202.

Kléman M, Williams CE, Costello MJ, Gulik-Krzywicki T. 1977. Defect structures in lyotropic smectic phases revealed by freeze-fracture electron microscopy. Philos Mag 35: 33-56.

Kostko AF, Cipriano BH, Pinchuk OA, Ziserman L, Anisimov MA, Danino D, Raghavan SR. 2005. Salt effects on the phase behavior, structure, and rheology of chromonic liquid crystals. J Phys Chem B 109: 19126-19133.

Leforestier A, Richter K, Livolant F, Dubochet J. 1996. Comparison of slam-freezing and highpressure freezing effects on the DNA cholesteric liquid crystalline structure. J Microsc 184: 4-13.

Leunissen JLM, Yi H. 2009. Self-pressurized rapid freezing (SPRF): a novel cryofixation method for specimen preparation in electron microscopy. J Microscopy 235: 25-35. 
Li XM, Mooney P, Zheng S, Booth C, Braunfeld MB, Gubbens S, Agard DA, Cheng YF. 2013. Electron counting and beam-induced motion correction enable near-atomic-resolution singleparticle cryo-EM. Nature Methods 10: 584-590.

Lydon J. 2010. Chromonic review. J Mater Chem 20: 10071-10099.

Lydon JE, Robinson DG. 1972. The structures of cholesteryl ester mesophases revealed by freeze-fracturing. Biochim Biophys Acta 260: 298-311.

Memmer R. 2002. Liquid crystal phases of achiral banana-shaped molecules: a computer simulation study. Liq Cryst 29: 483-496.

Meyer JC, Girit CO, Crommie MF, Zettl A. 2008. Imaging and dynamics of light atoms and molecules on graphene. Nature 454: 319-322.

Meyer RB. 1976. Structural problems in liquid crystal physics (Les Houches summer school in theoretical physics 1973). In: Molecular Fluids. Balian R, Weil G, editor. Gordon and Breach, New York, pp 273-373.

Mondain-Monval O. 2005. Freeze fracture TEM investigation in liquid crystals. Current Opinion in Colloid \& Interface Science 10: 250-255.

Nastishin YA, Liu H, Shiyanovskii SV, Lavrentovich OD, Kotko AF, Anisimov MA. 2004. Pretransitional fluctuations in the isotropic phase of a lyotropic chromonic liquid crystal. Phys Rev E 70: 051705.

Panov VP, Nagaraj M, Vij JK, Panarin YP, Kohlmeier A, Tamba MG, Lewis RA, Mehl GH. 2010. Spontaneous periodic deformations in nonchiral planar-aligned bimesogens with a nematic-nematic transition and a negative elastic constant. Phys Rev Lett 105: 167801.

Park HS, Lavrentovich OD. 2012. Lyotropic chromonic liquid crystals: emerging applications. In: Liquid Crystal Beyond Displays: Chemistry, Physics, and Applications. Li Q, editor. John Wiley \& Sons, Inc., Hoboken, New Jersey, pp 449-484.

Park HS, Kang SW, Tortora L, Kumar S, Lavrentovich OD. 2011. Condensation of selfassembled lyotropic chromonic liquid crystal sunset yellow in aqueous solutions crowded with polyethylene glycol and doped with salt. Langmuir 27: 4164-4175.

Pierron J, Boudet A, Sopena P. 1995. Cholesteric textures observed by transmission electron microscopy in diffraction contrast. Liq Cryst 19: 257-267.

Richter K. 1994. High-density morphologies of ice in high-pressure frozen biological specimens. Ultramicroscopy 53: 237-249. 
Rizwan SB, Dong YD, Boyd BJ, Rades T, Hook S. 2007. Characterisation of bicontinuous cubic liquid crystalline systems of phytantriol and water using cryo field emission scanning electron microscopy (cryo FESEM). Micron 38: 478-485.

Ruths M, Steinberg S, Israelachvili JN. 1996. Effects of confinement and shear on the properties of thin films of thermotropic liquid crystal. Langmuir 12: 6637-6650.

Severs NJ. 2007. Freeze-fracture electron microscopy. Nature Protocols 2: 547-576.

Shamid SM, Dhakal S, Selinger JV. 2013. Statistical mechanics of bend flexoelectricity and the twist-bend phase in bent-core liquid crystals. Phys Rev E 87: 052503.

Strunk K, Wang K, Ke D, Gray JL, and Zhang P. 2012. Thinning of large mammalian cells for cryo-TEM characterization by cryo-FIB milling. J Microsc 247:220-227.

Studer D, Humbel BM, Chiquest M. 2008. Electron microscopy of high pressure frozen samples: bridging the gap between cellular ultrastructure and atomic resolution. Histochem Cell Biol 130: 877-889.

Studer D, Graber W, Al-Amoudi A, Eggli P. 2001. A new approach for cryofixation by highpressure freezing. J Microsc 203: 285-294.

Tortora L, Park HS, Kang SW, Savaryn V, Hong SH, Kaznatcheev K, Finotello D, Sprunt S, Kumar S, Lavrentovich OD. 2010. Self-assembly, condensation, and order in aqueous lyotropic chromonic liquid crystals crowded with additives. Soft matter 6: 4157-5167.

Voigt-Martin IG, Durst H. 1987. Structure analysis of side chain liquid crystal polymer films by means of electron microscopy. Liq Cryst 2: 585-600.

Voigt-Martin IG, Garbella RW, Schumacher M. 1992. Structure and defects in discotic crystals and liquid crystals as revealed by electron diffraction and high-resolution electron microscopy. Macromolecules 25: 961-971.

Weissflog W, Baumeister U, Tamba MG, Pelzl G, Kresse H, Friedemann R, Hempel G, Kurz R, Roos M, Merzweiler K, Jakli A, Zhang C, Diorio N, Stannarius R, Eremin A, Kornek U. 2012. Unexpected liquid crystalline behaviour of three-ring bent-core mesogens: bis(4-subst.-phenyl) 2-methyl-iso-phthalates. Soft Matter 8: 2671-2685.

Yashima E. 2010. Synthesis and structure determination of helical polymers. Polymer Journal 42: 3-16. 
Zanchetta G, Nakata M, Buscaglia M, Bellini T, Clark NA. 2008. Phase separation and liquid crystallization of complementary sequences in mixtures of nanoDNA oligomers. Proc Natl Acad Sci USA 105: 1111-1117.

Zhang C, Gao M, Diorio N, Weissflog W, Baumeister U, Sprunt S, Gleeson JT, Jákli A. 2012. Direct observation of smectic layers in thermotropic liquid crystals. Phys Rev Lett 109: 107802.

Zhang PJ, Borgnia MJ, Mooney P, Shi D, Pan M, O’Herron P, Mao A, Brogan D, Milne JLS, Subramaniam S. 2003. Automated image acquisition and processing using a new generation of $4 \mathrm{~K} \times 4 \mathrm{~K}$ CCD cameras for cryo electron microscopic studies of macromolecular assemblies. $\mathrm{J}$ Structural Biology 143: 135-144.

Zhou S, Nastishin YA, Omelchenko MM, Tortora L, Nazarenko VG, Boiko OP, Ostapenko T, $\mathrm{Hu}$ T, Almasan CC, Sprunt SN, Gleeson JT, Lavrentovich OD. 2012. Elasticity of lyotropic chromonic liquid crystals probed by director reorientation in a magnetic field. Phys Rev Lett 109: 037801 .

Zhu G, Jiang Y, Huang W, Zhang H, Lin F, Jin C. 2013. Atomic resolution liquid-cell transmission electron microscopy investigations of the dynamics of nanoparticles in ultrathin liquids. Chem Commun 49: 10944-10946.

Zuo JM, Vartanyants I, Gao M, Zhang R, Nagahara LA. 2003. Atomic resolution imaging of a carbon nanotube from diffraction intensities. Science 300: 1419-1421.

Zuo JM, Zhang J, Huang W, Ran K, Jiang B. 2011. Combining real and reciprocal space information for aberration free coherent electron diffractive imaging. Ultramicroscopy 111: 817823. 
Figure Legends:

Figure 1. Molecular structures and names (full and short) of the LC materials used in this paper.

Figure 2. TEM specimen preparation routines for thermotropic LCs (TLC) (a-c) and lyotropic LCs (LLC) (d-f). (a) Plunge freezing of supported and suspended TLC thin films for cryo-TEM. (b) Cryo-sectioning of plunge frozen "bulk" TLC for cryo-TEM. (c) Freeze fracture of plunge frozen "bulk" TLC for FFTEM. (d) Plunge freezing of LLC thin film for cryo-TEM. (e) Vitreous sectioning of high pressure frozen LLC for cryo-TEM. (f) Freeze fracture of frozen (plunge freezing or high pressure freezing) LLC for replica TEM. The scale bars in the schematics roughly demonstrate the feature sizes.

Figure 3. (a) A cryo-TEM image revealing domain structure in a $3 \mathrm{RBC}-\mathrm{S}$ thin film quenched at $87^{\circ} \mathrm{C}$. The inset is the corresponding FFT pattern. The domains are numbered for convenience. (b) A magnified image of the local region identified by a dashed square in domain I of Fig. 3a. The inset shows a schematic of the molecule arrangement in the proposed SmA layer structure, and the arrow indicates the layer normal. Note that the molecules can rotate freely around the long axis at the absence of external fields. (c) A magnified image and its corresponding FFT pattern of the overlapped area of domains I and III (Fig. 3a).

Figure 4. (a) A typical cryo-TEM image showing the smectic nanoclusters in 3RBC-N nematic thin films. The narrow solid arrows point out a few overlapping clusters, while the hollow arrows denote assemblies of side-by-side clusters. The upper inset is the corresponding FFT pattern. The lower insets are the magnified views of the $30 \mathrm{~nm} \times 30 \mathrm{~nm}$ junction areas marked by squares. An extra half layer is pointed out by a solid arrow. (b) A representative region of smectic clusters with preferred orientation. (c) A rare local area with a large smectic assembly over 40 layers. (d) - (f) Magnified images of representative complex assemblies. The junctions are marked by hollow arrows, and the extra half layers by solid arrows.

Figure 5. (a) - (e) Cryo-TEM results of DSCG lyotropic chromonic LCs prepared by the "thin film" approach (plunge frozen specimens). (a) A typical image of $6.2 \%$ DSCG. (b) A typical image of 15\% DSCG. (c) Corresponding FFT pattern of the nematic structure shown in Fig. 5b. (d) A magnified image of the marked local area in Fig. 5a. (e) An image of the aggregates perpendicular to the thin film surface observed in 15\% DSCG. (f) and (g) CEMOVIS images of nematic regions in $15 \%$ DSCG with $10 \%$ dextran. The hollow arrow in $(\mathrm{g})$ points out a domain of aggregates perpendicular to the specimen surface.

Figure 6. Cryo-TEM images of SSY ( $\sim 65 \%$ water) lyotropic LCs using thin film plunge freezing (a) and bulk high pressure freezing and cryo-sectioning (b). 
Figure 7. (a) Schematics of molecular arrangement in $\mathrm{N}_{\mathrm{tb}}$ phase: molecules following a spiral (left) and molecules filling a small space (right). (b) A low magnification FFTEM image of $\mathrm{CB} 7 \mathrm{CB}$ quenched at $95^{\circ} \mathrm{C}$, showing layered structure. Domains I and II are corresponding to parallel and oblique fractures, respectively. The inset magnifies the enclosed area with slightly curved layers. (c) Magnified image of Domain I shown in (b) and the corresponding FFT pattern (the upper inset). The arrows point at the column boundaries in the image and the diffuse scattering perpendicular to the layer normal in the FFT pattern. The lower inset is a low-pass filtered gradient image of the marked rectangle area. The inset image is rotated for convenience and some of the boundaries are indicated by short lines at the top of the image. (d) and (e) FFTEM images showing Bouligand arches observed in oblique fracture domains. The wide and narrow arches are identified by arrows in (d). (f) and (g) FFTEM images of domains with reduced pitch near the domain boundaries.

Figure 8. (a) and (b) Typical cryo-TEM images from suspended thin films of pure CB7CB and CB7CB doped with right-handed limonene respectively. The inset in (a) shows a typical FFTEM image of a cluster of column-like circular features, which may be the top view of the columns in FFTEM as the fracture is perpendicular to the helix axis.

Figure 9. (a) A cryo-TEM image of a CB7CB thin layer supported by plasma-treated continuous carbon film. The upper inset image magnifies the marked local area, showing the sharp dots and lines inside the columns. The lower inset is a schematic model of the formation of the sharp dots and lines. (b) A cryo-TEM image of a CB7CB droplet on untreated continuous carbon film. (c) A cryo-TEM image of 3RBC-N thin film with a gradual increase in thickness from the film edge to the inside. The inset image magnifies the marked thick area (>500 nm in thickness). (d) Intensity profile along the line drawn in Fig. 9c, showing the variation of the thickness. The inset is an intensity profile along the line in the inset of Fig. 9c, demonstrating the modulated intensity of the layered cluster along the layer normal. (e) A cryo-TEM image of a thin part of the 3RBC-N thin film with very narrow smectic clusters. (f) A cryo-TEM image of a plunge-frozen DSCG thin film. The holes in this area are relatively small. The lacey carbon frame and several bundles of columnar aggregates perpendicular to the liquid surface are marked. The area marked by a dashed square is corresponding to the area in Fig. $5 \mathrm{~d}$.

Figure 10. (a) A TEM image showing the lattice of Au nanoparticles embedded in 5CB. The marked spacings are corresponding to Au (111) and (200) planes which have spacings of $2.36 \AA$ and $2.04 \AA$ in bulk Au, respectively. (b) A bright-field TEM image of Au nanoparticles in a thick area of a 5CB thin film. (c) A STEM Z-contrast image of Au nanoparticles (AuNP) in a thick area of $5 \mathrm{CB}$ film, demonstrating greatly enhanced contrast compared to the bright-field image. (d) EDS spectra of an individual Au nanoparticle and the nearby 5CB substrate taken by scanning 
the focused electron beam in a nanometer-sized box to reduce the beam damage and correct the specimen drift.

Table 1 A brief comparison of different TEM specimen preparation routines for LC materials.

\begin{tabular}{|c|c|c|c|c|c|c|c|}
\hline & \multicolumn{3}{|c|}{ Thermotropic } & \multicolumn{4}{|c|}{ Lyotropic } \\
\hline & Thin & Ultramicrotomy & FFTEM & Thin & Ultramicrotomy & FFT & EM \\
\hline $\begin{array}{l}\text { Freezing } \\
\text { technique }\end{array}$ & Plunge & Plunge & Plunge & Plunge & High pressure & Plunge & $\begin{array}{c}\text { High } \\
\text { pressure }\end{array}$ \\
\hline $\begin{array}{l}\text { Difficulty of } \\
\text { specimen } \\
\text { preparation }\end{array}$ & Low & High & Medium & Low & High & Medium & High \\
\hline $\begin{array}{l}\text { Complication } \\
\text { during } \\
\text { freezing }\end{array}$ & Low & Medium & Medium & Low & Medium & High & Medium \\
\hline $\begin{array}{c}\text { Consumed } \\
\text { material }\end{array}$ & $<<1 \mu \mathrm{l}$ & A few $\mu l$ & $\begin{array}{c}\text { A few } \\
\mu l\end{array}$ & $\begin{array}{c}\text { A few } \\
\mu l\end{array}$ & A few $\mu l$ & $\begin{array}{c}\text { A few } \\
\mu l\end{array}$ & $\begin{array}{c}\text { A few } \\
\mu l\end{array}$ \\
\hline $\begin{array}{l}\text { Process } \\
\text { induced } \\
\text { artifacts }\end{array}$ & Low & Medium & High & Low & Medium & High & High \\
\hline Cryo-TEM & Yes & Yes & No & Yes & Yes & No & No \\
\hline $\begin{array}{l}\text { Radiation } \\
\text { damage }\end{array}$ & High & High & Low & High & High & Low & Low \\
\hline $\begin{array}{c}\text { Surface } \\
\text { effect }\end{array}$ & Strong & Weak & Weak & Medium & Weak & $\begin{array}{l}\text { Weak- } \\
\text { medium }\end{array}$ & Weak \\
\hline Resolution & High & High & Medium & High & High & Low & Medium \\
\hline
\end{tabular}


Figure 1: Molecular structures and names (full and short) of the LC materials used in this paper. $259 \times 566 \mathrm{~mm}(300 \times 300 \mathrm{DPI})$ 
(a)

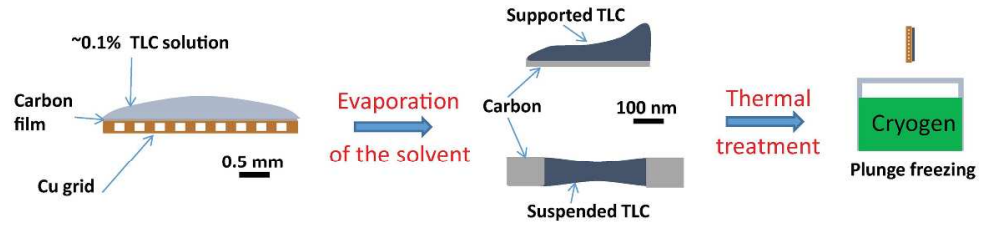

(b)
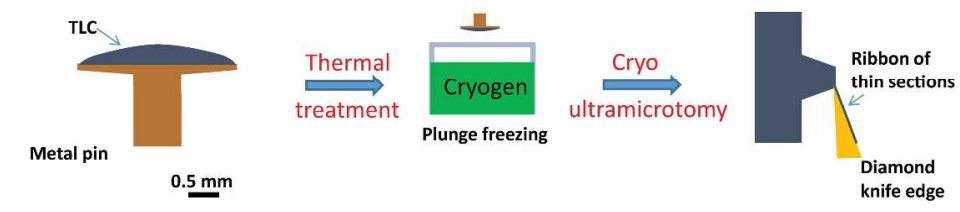

(c)
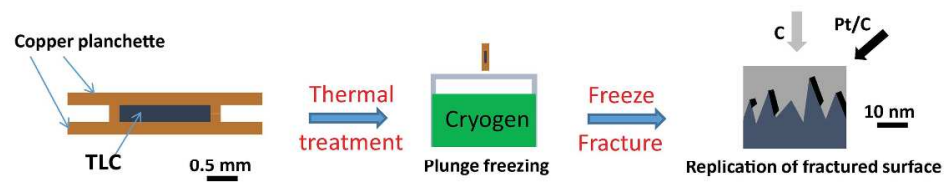

(e)

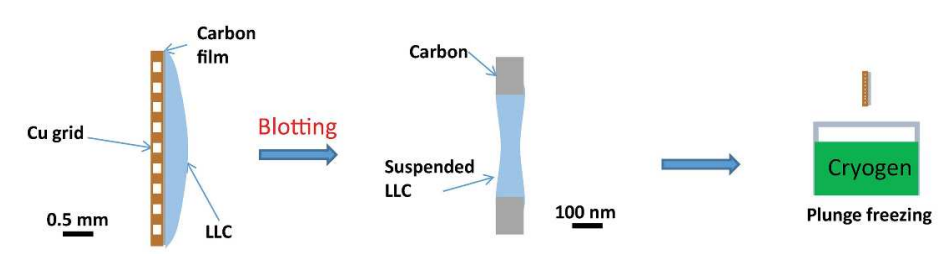

(d)

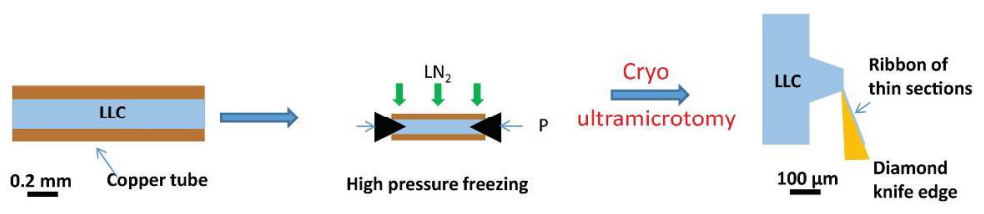

(f)

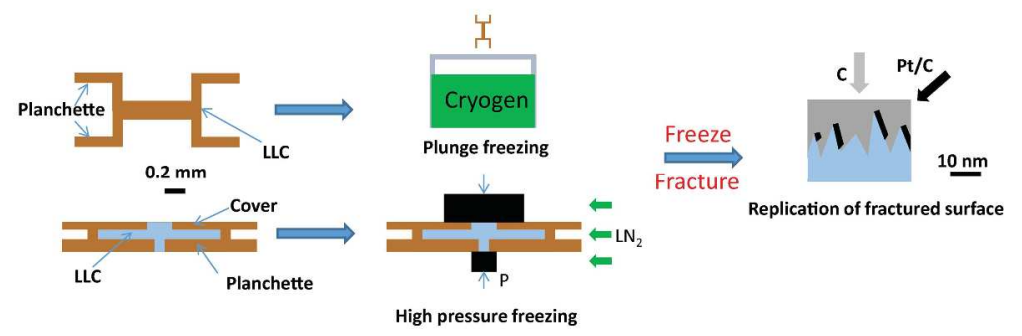

Figure 2: TEM specimen preparation routines for thermotropic LCs (TLC) (a-c) and lyotropic LCs (LLC) (d-f). (a) Plunge freezing of supported and suspended TLC thin films for cryo-TEM. (b) Cryo-sectioning of plunge frozen "bulk" TLC for cryo-TEM. (c) Freeze fracture of plunge frozen "bulk" TLC for FFTEM. (d) Plunge freezing of LLC thin film for cryo-TEM. (e) Vitreous sectioning of high pressure frozen LLC for cryo-TEM. (f) Freeze fracture of frozen (plunge freezing or high pressure freezing) LLC for replica TEM. The scale bars in the schematics roughly demonstrate the feature sizes.

$$
251 \times 357 \mathrm{~mm} \text { (300 x } 300 \text { DPI) }
$$




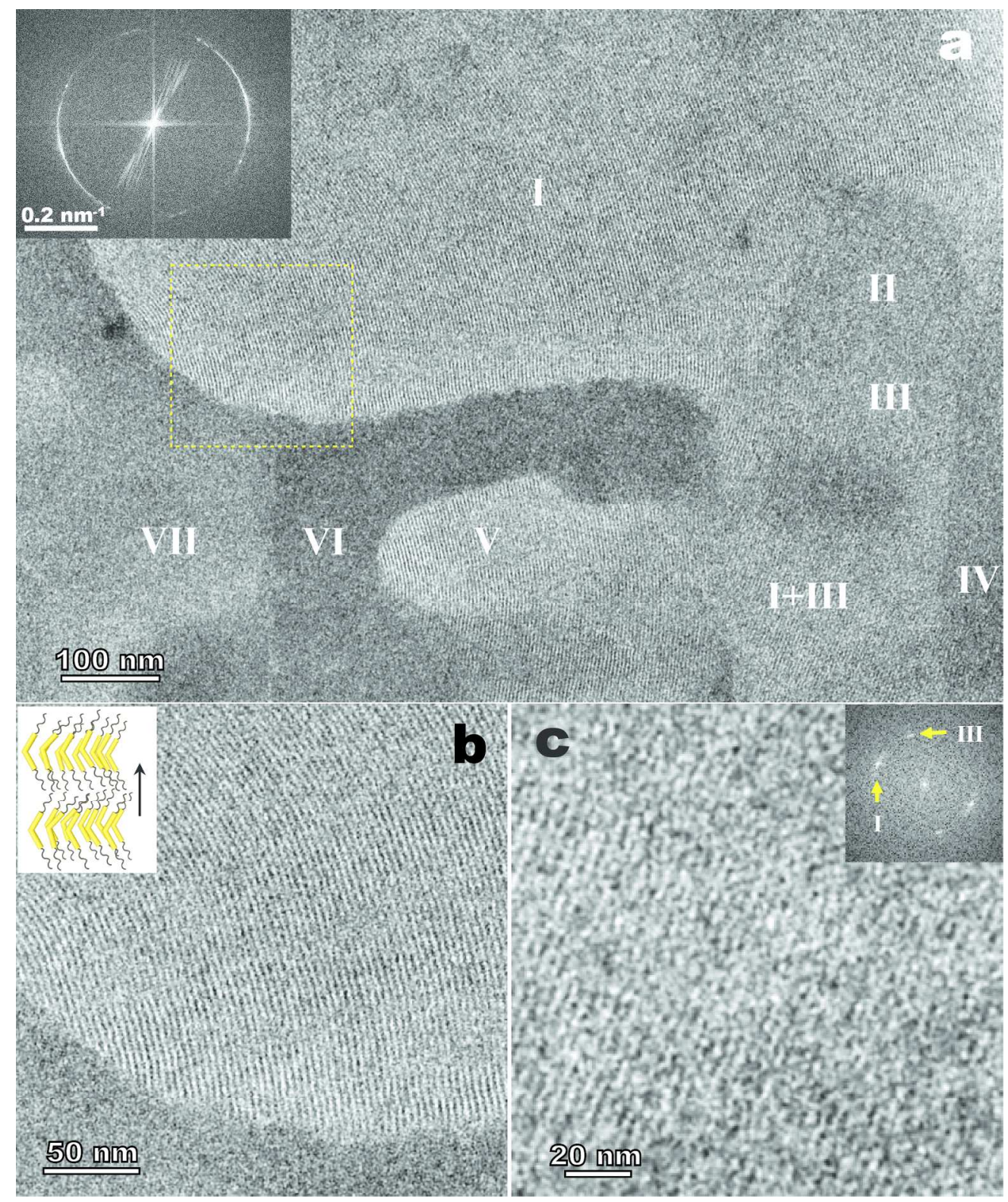

Figure 3: (a) A cryo-TEM image revealing domain structure in a $3 R B C-S$ thin film quenched at $87^{\circ} \mathrm{C}$. The inset is the corresponding FFT pattern. The domains are numbered for convenience. (b) A magnified image of the local region identified by a dashed square in domain I of Fig. 3a. The inset shows a schematic of the molecule arrangement in the proposed SmA layer structure, and the arrow indicates the layer normal. Note that the molecules can rotate freely around the long axis at the absence of external fields. (c) A magnified image and its corresponding FFT pattern of the overlapped area of domains I and III (Fig. 3a). $177 \times 212 \mathrm{~mm}(300 \times 300 \mathrm{DPI})$ 


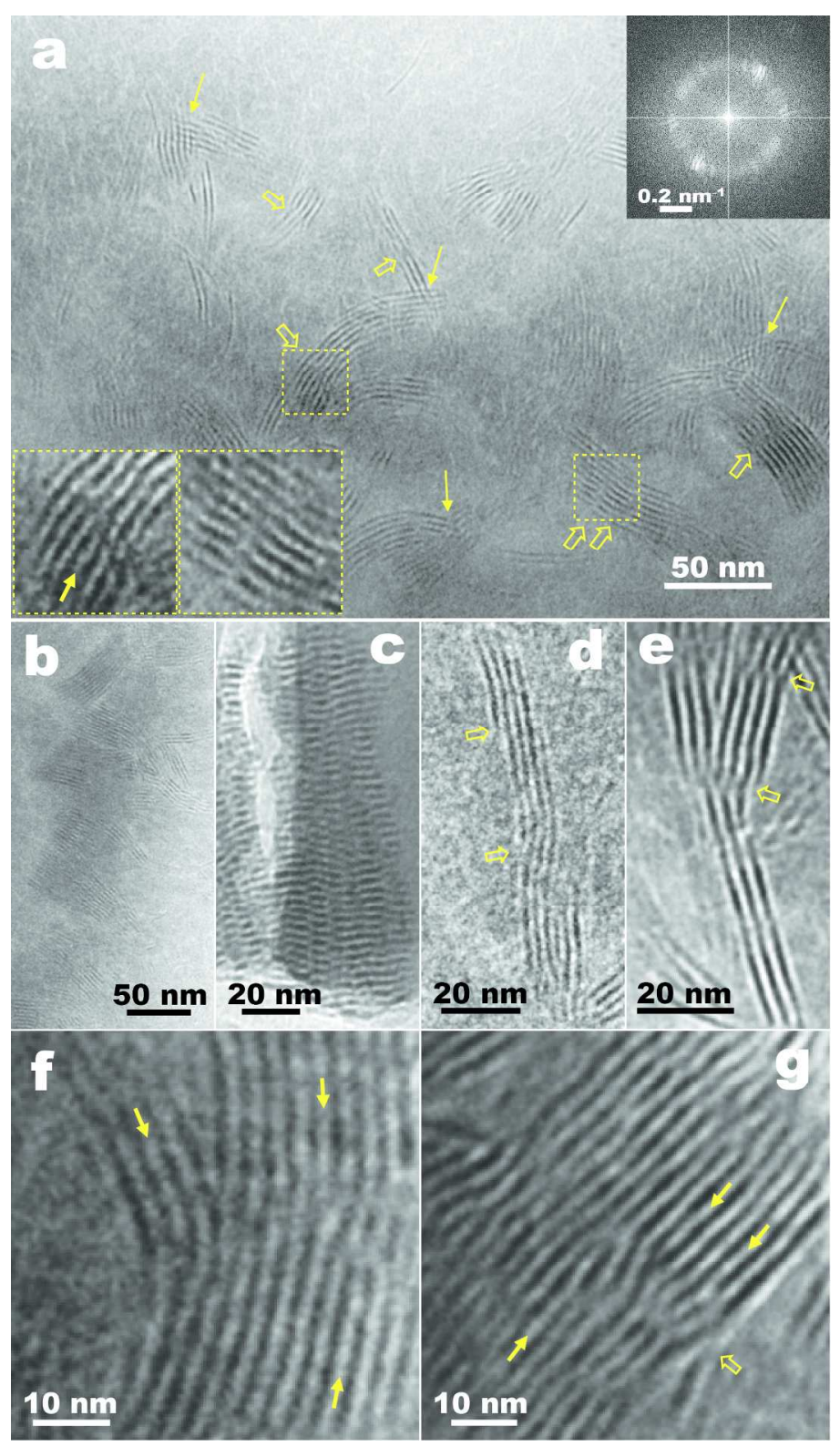

Figure 4: (a) A typical cryo-TEM image showing the smectic nanoclusters in 3RBC-N nematic thin films. The narrow solid arrows point out a few overlapping clusters, while the hollow arrows denote assemblies of sideby-side clusters. The upper inset is the corresponding FFT pattern. The lower insets are the magnified views of the $30 \mathrm{~nm} \times 30 \mathrm{~nm}$ junction areas marked by squares. An extra half layer is pointed out by a solid arrow.

(b) A representative region of smectic clusters with preferred orientation. (c) A rare local area with a large smectic assembly over 40 layers. (d) - (f) Magnified images of representative complex assemblies. The junctions are marked by hollow arrows, and the extra half layers by solid arrows. $142 \times 246 \mathrm{~mm}(300 \times 300 \mathrm{DPI})$ 


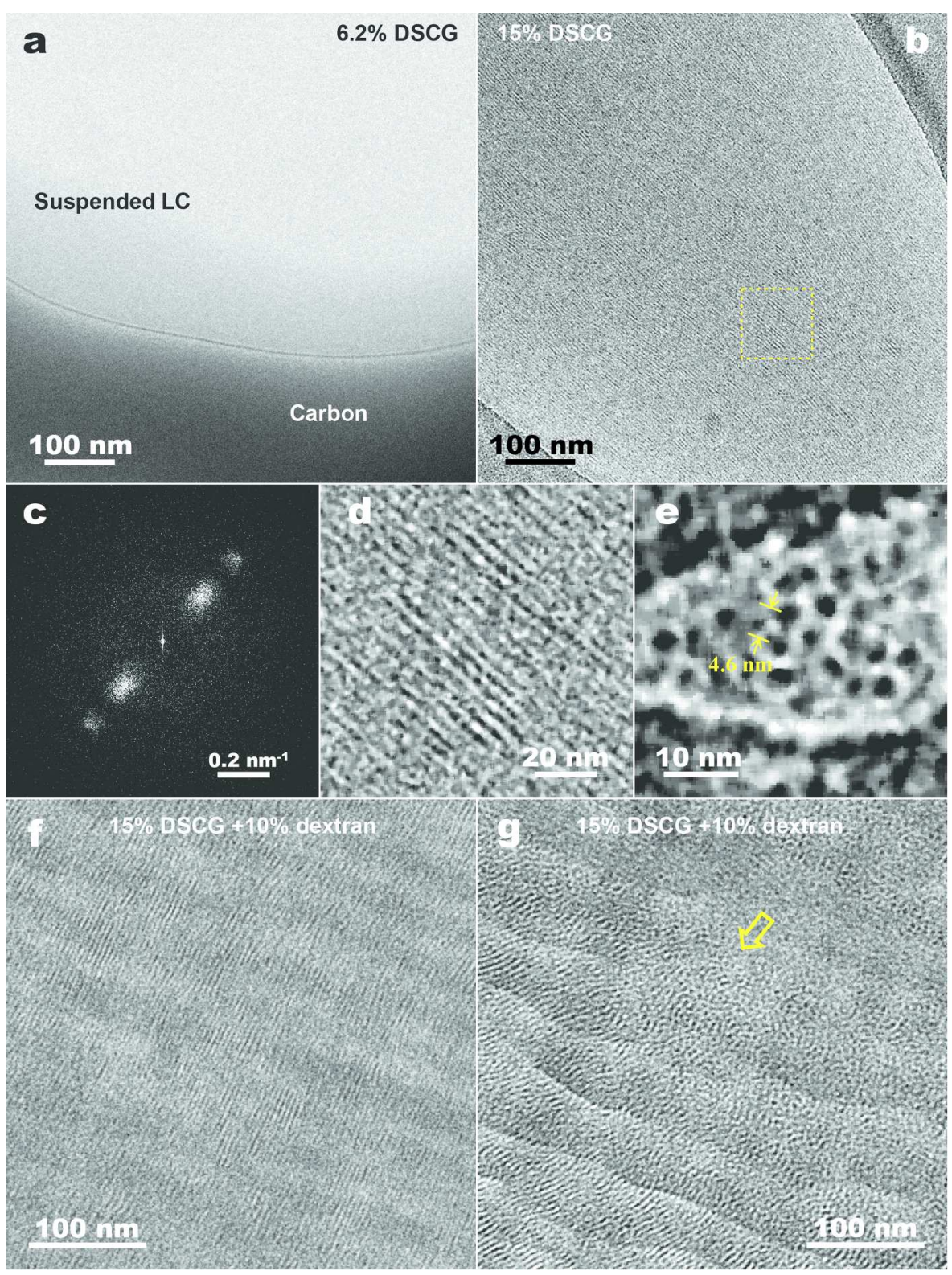

Figure 5: (a) - (e) Cryo-TEM results of DSCG lyotropic chromonic LCs prepared by the "thin film" approach (plunge frozen specimens). (a) A typical image of $6.2 \%$ DSCG. (b) A typical image of $15 \%$ DSCG. (c) Corresponding FFT pattern of the nematic structure shown in Fig. 5b. (d) A magnified image of the marked local area in Fig. 5a. (e) An image of the aggregates perpendicular to the thin film surface observed in $15 \%$ DSCG. (f) and (g) CEMOVIS images of nematic regions in 15\% DSCG with $10 \%$ dextran. The hollow arrow in (g) points out a domain of aggregates perpendicular to the specimen surface. $177 \times 236 \mathrm{~mm}(300 \times 300 \mathrm{DPI})$ 


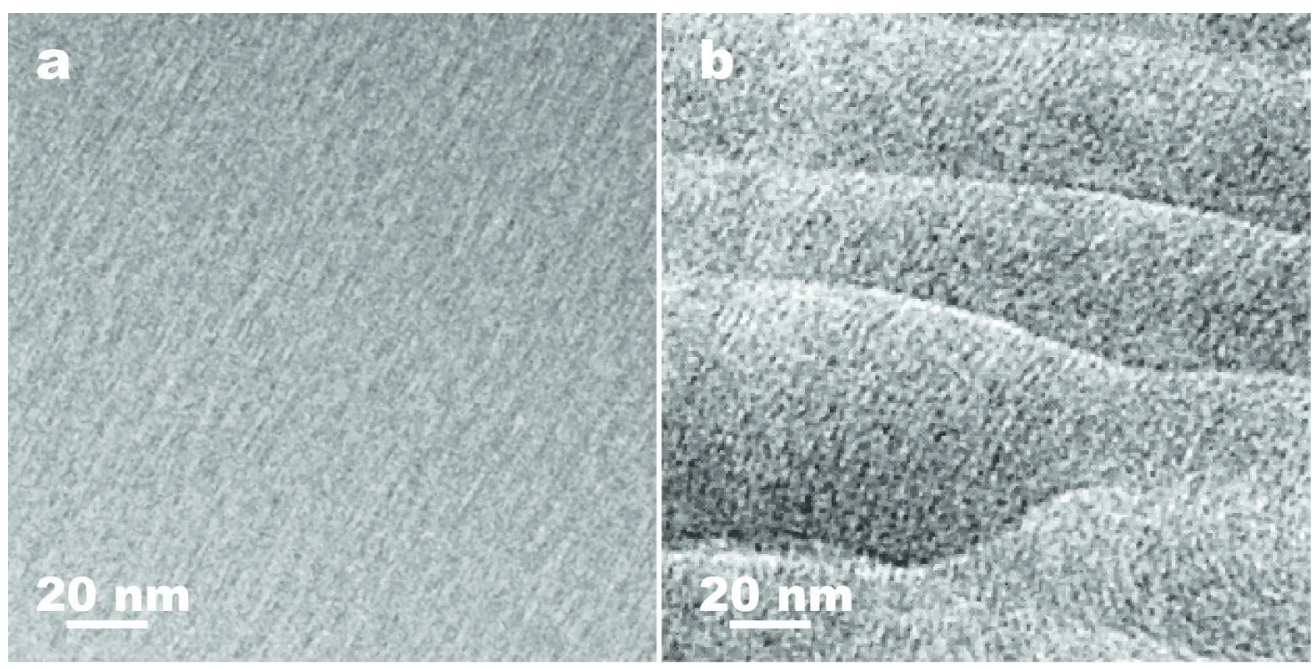

Figure 6: Cryo-TEM images of SSY ( $65 \%$ water) lyotropic LCs using thin film plunge freezing (a) and bulk high pressure freezing and cryo-sectioning (b). $177 \times 88 \mathrm{~mm}(300 \times 300$ DPI $)$ 

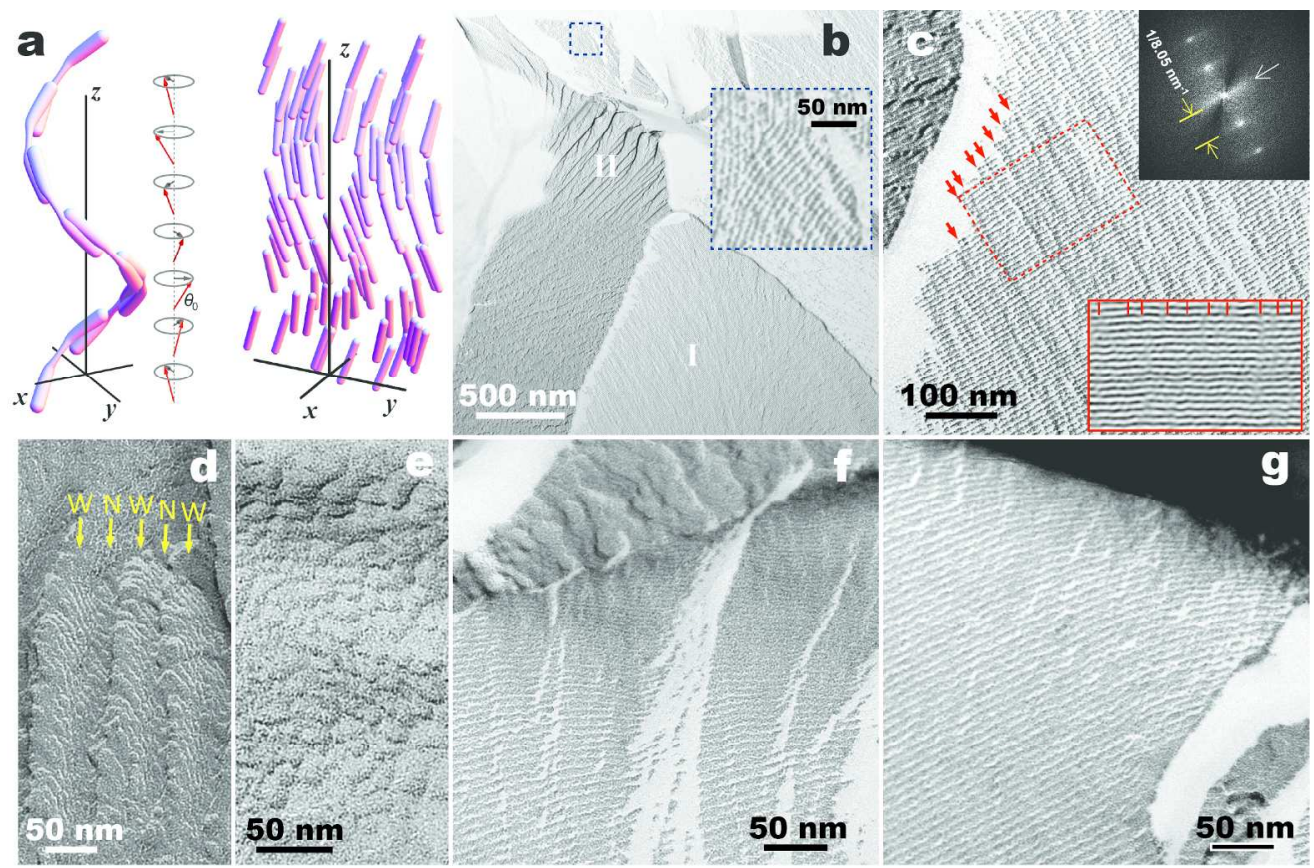

Figure 7: (a) Schematics of molecular arrangement in Ntb phase: molecules following a spiral (left) and molecules filling a small space (right). (b) A low magnification FFTEM image of CB7CB quenched at $95^{\circ} \mathrm{C}$, showing layered structure. Domains I and II are corresponding to parallel and oblique fractures, respectively. The inset magnifies the enclosed area with slightly curved layers. (c) Magnified image of Domain I shown in (b) and the corresponding FFT pattern (the upper inset). The arrows point at the column boundaries in the image and the diffuse scattering perpendicular to the layer normal in the FFT pattern. The lower inset is a low-pass filtered gradient image of the marked rectangle area. The inset image is rotated for convenience and some of the boundaries are indicated by short lines at the top of the image. (d) and (e) FFTEM images showing Bouligand arches observed in oblique fracture domains. The wide and narrow arches are identified by arrows in (d). ( $f$ ) and (g) FFTEM images of domains with reduced pitch near the domain boundaries.

\section{$215 \times 141 \mathrm{~mm}(300 \times 300 \mathrm{DPI})$}




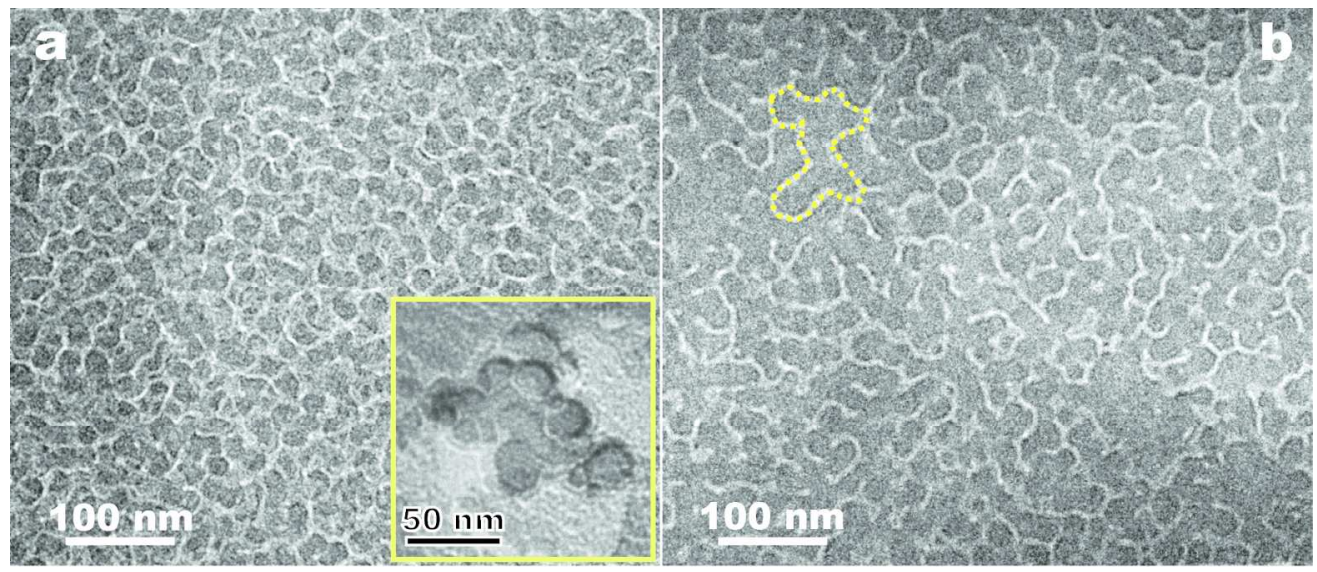

Figure 8: (a) and (b) Typical cryo-TEM images from suspended thin films of pure CB7CB and CB7CB doped with right-handed limonene respectively. The inset in (a) shows a typical FFTEM image of a cluster of column-like circular features, which may be the top view of the columns in FFTEM as the fracture is perpendicular to the helix axis. $176 \times 75 \mathrm{~mm}(300 \times 300 \mathrm{DPI})$ 


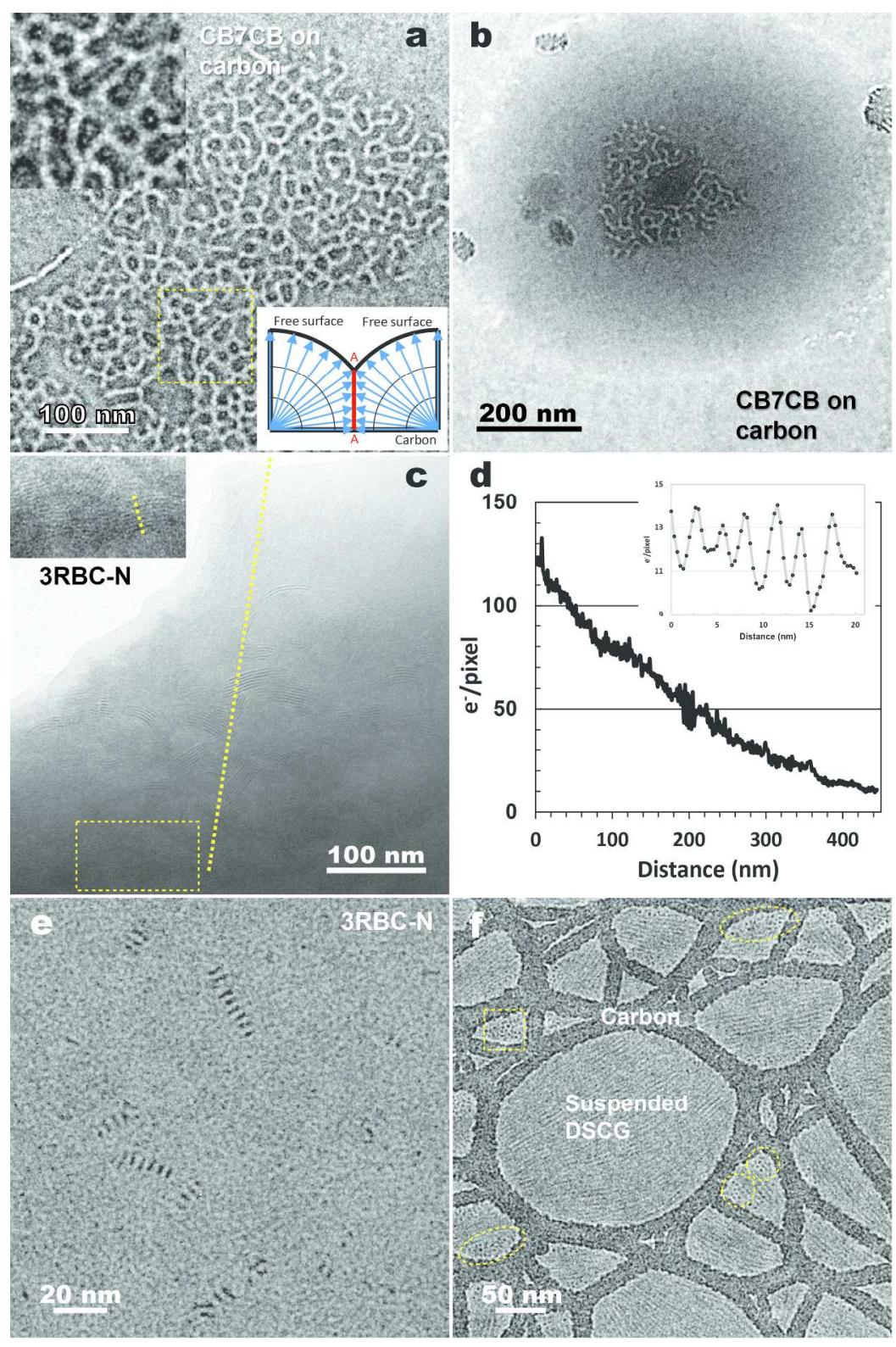

Figure 9: (a) A cryo-TEM image of a CB7CB thin layer supported by plasma-treated continuous carbon film. The upper inset image magnifies the marked local area, showing the sharp dots and lines inside the columns. The lower inset is a schematic model of the formation of the sharp dots and lines. (b) A cryo-TEM image of a CB7CB droplet on untreated continuous carbon film. (c) A cryo-TEM image of 3RBC-N thin film with a gradual increase in thickness from the film edge to the inside. The inset image magnifies the marked thick area (> $500 \mathrm{~nm}$ in thickness). (d) Intensity profile along the line drawn in Fig. 9c, showing the variation of the thickness. The inset is an intensity profile along the line in the inset of Fig. $9 \mathrm{c}$, demonstrating the modulated intensity of the layered cluster along the layer normal. (e) A cryo-TEM image of a thin part of the 3RBC-N thin film with very narrow smectic clusters. (f) A cryo-TEM image of a plungefrozen DSCG thin film. The holes in this area are relatively small. The lacey carbon frame and several bundles of columnar aggregates perpendicular to the liquid surface are marked. The area marked by a dashed square is corresponding to the area in Fig. $5 \mathrm{~d}$. $198 \times 266 \mathrm{~mm}(300 \times 300 \mathrm{DPI})$ 


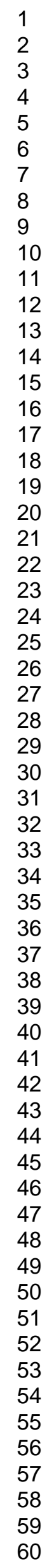


Figure 10. (a) A TEM image showing the lattice of Au nanoparticles embedded in 5CB. The marked spacings are corresponding to Au (111) and (200) planes which have spacings of $2.36 \AA$ and $2.04 \AA$ in bulk Au, respectively. (b) A bright-field TEM image of Au nanoparticles in a thick area of a 5CB thin film. (c) A STEM Z-contrast image of Au nanoparticles (AuNP) in a thick area of 5CB film, demonstrating greatly enhanced contrast compared to the bright-field image. (d) EDS spectra of an individual Au nanoparticle and the nearby $5 \mathrm{CB}$ substrate taken by scanning the focused electron beam in a nanometer-sized box to reduce the beam damage and correct the specimen drift. $177 \times 176 \mathrm{~mm}(300 \times 300 \mathrm{DPI})$ 